\title{
Minimization of Tree Pattern Queries with Constraints
}

\author{
Ding Chen and Chee-Yong Chan \\ Dept. of Computer Science, School of Computing \\ National University of Singapore \\ \{chending, chancy\}@comp.nus.edu.sg
}

\begin{abstract}
Tree pattern queries (TPQs) provide a natural and easy formalism to query tree-structured XML data, and the efficient processing of such queries has attracted a lot of attention. Since the size of a TPQ is a key determinant of its evaluation cost, recent research has focused on the problem of query minimization using integrity constraints to eliminate redundant query nodes; specifically, TPQ minimization has been studied for the class of forward and subtype constraints (FT-constraints). In this paper, we explore the TPQ minimization problem further for a richer class of FBST-constraints that includes not only FT-constraints but also backward and sibling constraints. By exploiting the properties of minimal queries under FBST-constraints, we propose efficient algorithms to both compute a single minimal query as well as enumerate all minimal queries. In addition, we also develop more efficient minimization algorithms for the previously studied class of FT-constraints. Our experimental study demonstrates the effectiveness and efficiency of query minimization using FBST-constraints.
\end{abstract}

\section{Categories and Subject Descriptors}

H.2.4 [Database Management]: Systems - Query processing

\section{General Terms}

Algorithms, Performance

\section{Keywords}

XML, XPath, Tree pattern queries, Query minimization, Integrity constraints, Chase, Simulation

\section{INTRODUCTION}

Tree pattern queries (TPQs) provide a very natural and easy formalism to query XML data, and constitute a very useful and large fragment of queries expressible using XML query languages such as XPath [17] and XQuery [18]. Since the size of the query (in terms of the number of query steps) is a key determinant of its evaluation cost [9], there has been a lot of interest in the minimization

Permission to make digital or hard copies of all or part of this work for personal or classroom use is granted without fee provided that copies are not made or distributed for profit or commercial advantage and that copies bear this notice and the full citation on the first page. To copy otherwise, to republish, to post on servers or to redistribute to lists, requires prior specific permission and/or a fee.

SIGMOD'08, June 9-12, 2008, Vancouver, BC, Canada.

Copyright 2008 ACM 978-1-60558-102-6/08/06 ...\$5.00. of TPQs given knowledge of data constraints [19, 5, 14, 8]. The work in this area can be characterized along two main dimensions: the class of queries being supported (i.e., query fragment) and the types of constraints being considered. The various fragments of XPath queries explored so far can be denoted by $X P^{F}$ [8], where $F \subseteq\{/, / /,[], *\}$ represents the set of query features supported including child axis "/", descendant axis "I/", nested predicates "[]", and wildcards " $*$ ".

In terms of data constraints, besides the simplest case of query minimization without considering constraints, most of the work has focused primarily on forward constraints and subtype constraints $[5,14]$. There are two types of forward constraints, namely, required child $(R C)$ constraints and required descendant $(R D)$ constraints. An RC (RD resp.) constraint is of the form $x \rightarrow y(x \rightarrow y$ resp.) which states that for every element of type $x$, it has a child (proper descendant resp.) element of type $y$. A subtype constraint is of the form $x \leq y$ which states that every element of type $x$ is also of type $y$.

In this paper, we examine the minimization of TPQs for the query fragment $X P^{\{/, / /,[]\}}$with respect to a richer class of data constraints that include not only forward and subtype constraints but also backward and sibling constraints.

Backward constraints, which are the "opposites" of forward constraints, can be classified into two types, namely, required parent $(R P)$ constraints and required ancestor $(R A)$ constraints. An RP (RA resp.) constraint is of the form $x \leftarrow y$ ( $x \nleftarrow y$ resp.) which states that for every element of type $y$, it has a parent (proper ancestor resp.) element of type $x$. For an example of a RP constraint, if an element ' $b$ ' appears as a sub-element only for the element ' $a$ ', then we have $a \leftarrow b$. For an example of a RA constraint, if an element ' $d$ ' appears as a sub-element only for elements ' $b$ ' and ' $c$ ', and both elements ' $b$ ' and ' $c$ ' appear as sub-elements only for element ' $a$ ', then we have $a \nleftarrow d$.

A sibling constraint is of the form $a \stackrel{c}{a}$ b which states that for every element of type $a$, if it has a child element of type $c$, then the $a$ element must also have a child element of type $b$. Note that for a sibling constraint $a \stackrel{c}{\rightarrow} \rightarrow b$ to hold, it is not necessary to have $a \rightarrow b$ and $a \rightarrow c$. For example, the following are two possible DTD type definitions for an element ' $a$ ' that will result in the sibling constraint $a \stackrel{c}{\rightarrow} b:((c ?, b+) *, d)$ and $((b, c) \mid d)$.

Despite the fact that backward and sibling constraints have been largely neglected (with respect to query minimization), they are actually rather common in XML data. Table 1 compares the number of forward and backward constraints that are extracted from five different DTDs ${ }^{1}$. Observe that there are actually more backward constraints than forward constraints in these DTDs.

\footnotetext{
${ }^{1}$ Note that the number of forward and backward constraints indicated refer to the number of "basic" constraints that cannot be de-
} 


\begin{tabular}{|c||c|c||c|c|}
\hline \multirow{2}{*}{\multicolumn{1}{|c||}{ XML }} & \multicolumn{2}{c|}{ Forward } & \multicolumn{2}{c|}{ Backward } \\
\cline { 2 - 5 } Database & RC & RD & RP & RA \\
\hline \hline GraphML [7] & 0 & 0 & 6 & 5 \\
\hline DBLP [11] & 0 & 0 & 8 & 27 \\
\hline PSDML [1] & 26 & 0 & 57 & 8 \\
\hline XMark [15] & 57 & 0 & 57 & 14 \\
\hline Mondial [3] & 13 & 0 & 30 & 8 \\
\hline
\end{tabular}

Table 1: Forward \& Backward Constraints in XML DTDs

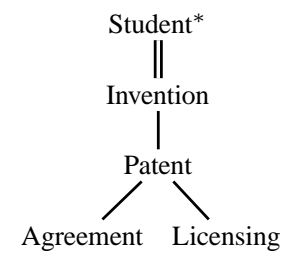

(a) $Q_{1}$

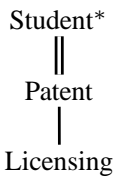

(d) $Q_{4}$

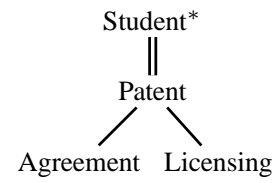

$\prod_{\text {Patent }}^{\text {Student* }}$

(c) $Q_{3}$

(b) $Q_{2}$

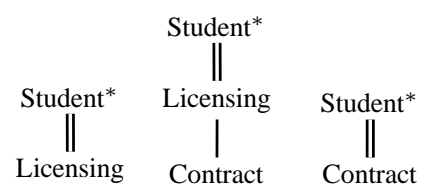

(e) $Q_{5}$

(f) $Q_{6}$

(g) $Q_{7}$
Figure 1: Minimization with Backward \& Sibling Constraints

Furthermore, as pointed out by Bex, et al. [6] and Hinkelman [10], XML schemas (including industry-level standards) are generally too loosely defined with respect to the data that they actually represent. This means that XML data sets generally satisfy more constraints than what are explicitly specified in their schemas. We conducted a simple study to identify the sibling constraints from the data sets maintained at the XML Data Repository [11]. We found that there are 121, 6, and 3 sibling constraints, respectively, in the DBLP, Protein Sequence Database, and Mondial data sets ${ }^{2}$, although there are only 0,1 and 0 sibling constraints specified in their corresponding DTDs respectively. As an example, in the DBLP data set, the 'phdthesis' element does not always have a 'publisher' sub-element. However, if a 'phdthesis' element has an 'isbn' sub-element, then the 'phdthesis' element must also have a 'publisher' sub-element. Although the sibling constraint phdthesis $\stackrel{i s b n}{\rightarrow}$ publisher is not explicitly captured by DBLP's DTD, this constraint is indeed satisfied by the DBLP data sets [11].

By considering a richer class of constraints, there are more opportunities for query minimization. As an example, consider the minimization of the TPQ $Q_{1}$ shown in Figure 1(a) w.r.t. the set of constraints $C=\{$ Patent $\stackrel{\text { Agreement }}{\rightarrow}$ Licensing, Patent $\stackrel{\text { Licensing }}{\rightarrow \rightarrow}$ Agreement, Patent $\leftarrow$ Licensing, Invention $\leftarrow$ Patent, Patent $\rightarrow$ Title, Licensing $\rightarrow$ Contract, Licensing $\leftarrow$ Contract $\}$. Each node in $Q_{1}$ represents an element type, and the special node that is marked with a ${ }^{*}$ (i.e., Student) represents the output node of the query. Thus $Q_{1}$ will return all Student elements that satisfy a set of requirements specified by the edges. A single (double) edge con-

rived from other constraints.

${ }^{2}$ Again here, we counted only the "basic" sibling constraints that cannot be derived from other constraints. necting two nodes represents a parent-child (ancestor-descendant) relationship. Thus, two of the requirements specified by $Q_{1}$ are that each Student element must have a descendant Invention element, and each Invention element must have a child Patent element. Observe that $Q_{1}$ cannot be further minimized using only the forward constraints in $C$. However, using the RP constraint Invention $\leftarrow$ Patent, $Q_{1}$ can be simplified to $Q_{2}$ (Figure 1(b)). Moreover, by applying the sibling constraint Patent $\stackrel{\text { Agreement }}{\rightarrow}$ Licensing, $Q_{2}$ can be further minimized to $Q_{3}$ (Figure 1(c)), which turns out to be a minimal query (w.r.t. $C$ ).

Query minimization using backward and/or sibling constraints is a more challenging problem due to two new properties of minimal queries under such constraints. First, the minimal query is not necessarily unique; and moreover, the minimal queries do not necessarily have the same size. Second, a minimal query can contain element types that are not present in the input query. In contrast, the minimal query (with respect to only forward and subtype constraints) is always unique, and the element types appearing in the minimal query is a subset of those in the input query [5, 14].

Referring again to the example in Figure $1, Q_{1}$ actually has three minimal queries: besides $Q_{3}, Q_{5}$ and $Q_{7}$ are also minimal queries of $Q_{1}$. Note that the sizes of $Q_{3}$ and $Q_{5}$ are different, and $Q_{7}$ contains the element type Contract that is absent in $Q_{1}$. The second minimal query $Q_{5}$ can be derived from $Q_{2}$ as follows: first, apply the sibling constraint Patent $\stackrel{\text { Licensing }}{-\rightarrow}$ Agreement to minimize $Q_{2}$ to $Q_{4}$; next, apply the RP constraint Patent $\leftarrow$ Licensing to simplify $Q_{4}$ to $Q_{5}$. The third minimal query $Q_{7}$ is obtained from $Q_{5}$ as follows: first, note that $Q_{5}$ is equivalent to $Q_{6}$ due to the RC constraint Licensing $\rightarrow$ Contract; next, note that $Q_{6}$ is equivalent to $Q_{7}$ due to the RP constraint Licensing $\leftarrow$ Contract.

For notational convenience, we use the letters F, B, S, and T to represent, respectively, the class of forward, backward, sibling, and subtype constraints. In addition, we use $\alpha$-constraints to denote the class of constraints of types in $\alpha$, where $\alpha \subseteq\{F, B, S, T\}$; braces and commas in $\alpha$ are omitted for simplicity.

The key results of our paper are summarized in Figure 2. Figure 2(a) compares the key properties of minimal queries (columns 2 to 4 ) for different classes of data constraints (column 1): our new results are indicated in rows 2 to 4 , while the results from previous work $[5,14]$ are indicated in row 1 . The lattice structure in Figure 2(b) summarizes the time-complexity of query minimization for $X P^{\{/, / /,[]\}}$under different constraint classes (represented by the lattice nodes), where the lattice edges represent the containment relationship between constraint classes. Specifically, the time complexity shown for each class is for computing one minimal query of an input query $Q$ w.r.t. a set of constraints $C$, where $n$ denotes the number of steps in $Q$, and $\Sigma$ denotes the set of distinct element types in $C$.

The rest of the paper is organized as follows. Section 2 covers background material, and related work is presented in Section 3. Section 4 introduces key concepts for query minimization. Section 5 presents properties of minimal queries under different classes of constraints. Section 6 presents efficient algorithms to generate a minimal query and to enumerate all the minimal queries under FBST-constraints. Section 7 presents a more efficient minimization algorithm for FT-constraints. Section 8 covers the minimization algorithms and results for the remaining subclasses of FBSTconstraints. Section 9 presents an experimental evaluation of our proposed algorithms. Finally, Section 10 concludes the paper.

\section{BACKGROUND}

Tree pattern queries. As illustrated in Figure 1, tree pattern 


\begin{tabular}{|c|c|c|c|}
\hline $\begin{array}{c}\text { Data } \\
\text { constraints }\end{array}$ & $\begin{array}{c}\text { Number of } \\
\text { minimal queries }\end{array}$ & $\begin{array}{l}\text { Element types in } \\
\text { minimal queries }\end{array}$ & $\begin{array}{l}\text { How do minimal } \\
\text { queries differ? }\end{array}$ \\
\hline $\mathrm{F} / \mathrm{FT}$ & One & $\begin{array}{l}\text { Subset of original } \\
\text { query's types }\end{array}$ & - \\
\hline FB / FBT & \multirow{2}{*}{$\begin{array}{l}\text { Possibly multiple minimal } \\
\text { queries of the same size }\end{array}$} & \multirow{3}{*}{$\begin{array}{l}\text { Can contain } \\
\text { element types that } \\
\text { are not present in } \\
\text { original query }\end{array}$} & ad-leaf nodes \\
\hline FS/FST & & & $p c$-leaf nodes \\
\hline FBS / FBST & $\begin{array}{c}\text { Possibly multiple minimal } \\
\text { queries of different sizes }\end{array}$ & & pclad-leaf nodes \\
\hline
\end{tabular}

(a) Properties of Minimal Queries

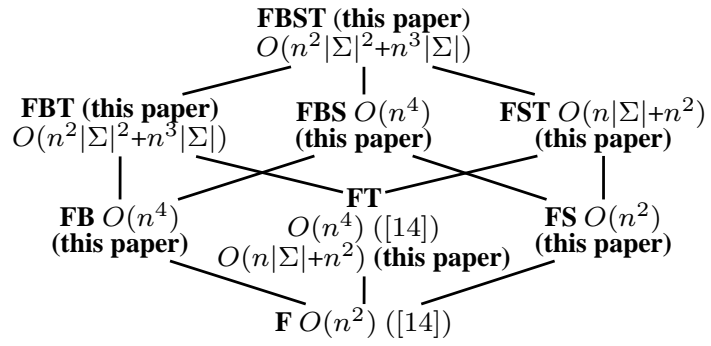

(b) Time Complexity of TPQ minimization (n = size of query, $\Sigma=$ set of element types in constraints)

Figure 2: Summary of Key Results $(\mathrm{F}=$ forward, $\mathrm{B}=$ backward, $\mathrm{S}=\operatorname{sibling}, \mathrm{T}=$ subtype $)$

queries (TPQs) are represented as trees, where the nodes of a TPQ $Q$ are labelled by element types from a finite alphabet $\Sigma$. The type of a node $u$ is denoted by $\tau(u)$, and the root node of $Q$ is denoted by $\operatorname{root}(Q)$. The size of $Q$, denoted by $|Q|$, refers to the number of nodes in $Q$. Each query $Q$ has a unique output node, denoted as $o p(Q)$, and its element type label is distinguished with a * mark. The nodes in $Q$ are connected by two types of edges: parent-child edges ( $p c$-edges) and ancestor-descendant edges ( $a d$-edges). Consider an edge $e=(u, v)$ with parent node $u$ and child node $v$. If $e$ is a $\alpha$-edge, where $\alpha \in\{p c, a d\}$, we say that $v$ is a $\alpha$-child of $u$ and $u$ is the $\alpha$-parent of $v$. Moreover, if $v$ is a leaf node, $v$ is also known as a $\alpha$-leaf node.

An embedding of a TPQ $Q$ onto a tree database $d b$ is defined as a mapping $\beta$ from the nodes of $Q$ to the nodes of $d b$ such that the following conditions are satisfied:

1. Preserve node types: for each node $u \in Q$, either $u$ and $\beta(u)$ are of the same type, or $\beta(u)$ is of a subtype of $u$;

2. Preserve $p c / a d$-edge relationships: if $v$ is a $p c$-child ( $a d$ child resp.) of $u$ in $Q$, then $\beta(v)$ is a child (descendant resp.) of $\beta(u)$ in $d b$.

The evaluation of a TPQ $Q$ on $d b$ requires finding all the embeddings of $Q$ in $d b$, and the answer to $Q$ is given by the set of database nodes $\beta(o p(Q))$.

Minimal queries. A node $u$ of a TPQ $Q$ is redundant if $u$ is a non-output node and the query obtained by deleting $u$ from $Q$ is equivalent to $Q$. Here, deleting a leaf node $u$ simply removes $u$ and its incident edge; while deleting an internal node $u$ requires removing $u$ and connecting $u$ 's parent node (if it exists) to each of $u$ 's child nodes with an $a d$-edge.

Given two TPQs $Q$ and $Q^{\prime}$, and a set of integrity constraints (ICs) $C, Q$ and $Q^{\prime}$ are equivalent w.r.t. $C$, denoted by $Q \equiv_{C} Q^{\prime}$, if and only if $Q$ and $Q^{\prime}$ have the same answer on all tree databases that satisfy $C$. $Q^{\prime}$ is a minimal query of $Q$ w.r.t. $C$ iff (1) $Q \equiv_{C} Q^{\prime}$ and (2) $Q \not_{C} Q^{\prime \prime}$ for every $Q^{\prime \prime}$ that is obtained by deleting some node(s) from $Q^{\prime}$.

Notations. In this paper, we use $\Sigma$ to denote the set of distinct element types in the constraints, and $n$ to denote the size of $Q$.

\section{RELATED WORK}

Sihem et al. were the first to study the TPQ minimization problem for the query fragment $X P^{\{/, / /,[]\}}$[5]. The state-of-the-art minimization algorithms for this fragment have time complexities of $O\left(n^{2}\right)$ and $O\left(n^{4}\right)$, respectively, for the case without constraints and the case with FT-constraints [14]. Moreover, for both cases, every TPQ has a unique minimal query $[5,14]$. To the best of our knowledge, TPQ minimization for $X P^{\{/, / /,[]\}}$using backward or sibling constraints has not been explored.

Query minimization for the full query fragment $X P^{\{/, / /,[], *\}}$ in the absence of constraints was shown by Flesca et al. to be NP-hard [8]. For fragment $X P^{\{/,[], *\}}$, query minimization in the absence of constraints has polynomial time complexity, and each XPath query has a unique minimal query [19].

A related direction is query containment $[12,13,20]$. The containment problem under DTDs for a smaller XPath fragment $X P^{\{/,[]\}}$ was shown to be coNP-complete [13].

\section{REASONING WITH CONSTRAINTS}

This section introduces the key concepts for TPQ minimization under FBST-constraints.

\subsection{Constraint Closure}

Given a set of FBST-constraints $C$, let closure $(C)$ denote the set of FBST-constraints that must hold w.r.t. $C$. The following set of inference rules (R1 to R22) can be used to compute closure $(C)$ as follows: first, initialize closure $(C)$ to be $C$, and then iteratively add new constraints that are generated by the rules to closure $(C)$ until no further rules can be added.

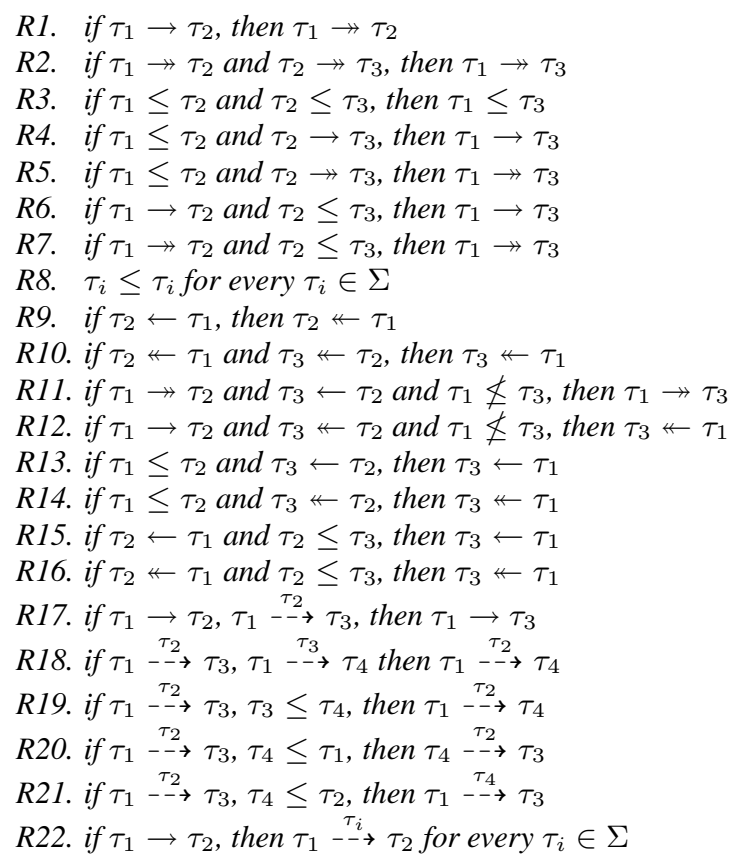


In each rule, $\tau_{1}, \tau_{2}, \tau_{3}$, and $\tau_{4}$ represent distinct element types. We write $\tau_{1} \not \leq \tau_{3}$ to mean that $\tau_{1}$ is not a subtype of $\tau_{3}$. Rules $\mathrm{R} 1$ to R8 were used earlier in [14] for query minimization with FT-constraints. Rules R9 to R16 are new rules to handle backward constraints, while rules R17 to R22 are new rules to handle sibling constraints.

Rule R11 follows from the tree structure property: if a node $u_{1}$ of type $\tau_{1}$ has a descendant $u_{2}$ of type $\tau_{2}$, and $u_{2}$ in turn has a parent $u_{3}$ of type $\tau_{3}$, then $u_{3}$ must be a descendant of $u_{1}$ provided that $\tau_{1}$ is not a subtype of $\tau_{3}$. Otherwise, if $\tau_{1} \leq \tau_{3}$, then $u_{3}$ may not be a descendant of $u_{1}$ as $u_{3}$ and $u_{1}$ can be the same node. A similar reasoning applies to rule R12. The rest of the rules are straight-forward.

\subsection{Constraint Graph}

The FBS-constraints in closure $(C)$ can be categorized into trivial and non-trivial constraints defined as follows. Every RC and $\mathrm{RP}$ constraint is non-trivial. An RD or RA constraint is trivial if it can be inferred from other constraints using rules that do not involve subtype constraints; otherwise it is non-trivial. A sibling constraint is trivial if it can be inferred using rule R22; otherwise it is non-trivial.

Constraint graph. The non-trivial FBS-constraints in closure $(C)$ can be represented succinctly by a constraint graph, denoted by $G_{C}=\left(V_{C}, E_{C}\right)$, where each node in $V_{C}$ represents some element type in $\Sigma$ and each edge in $E_{C}$ represents a non-trivial constraint. Specifically, if $\tau_{1} \rightarrow \tau_{2}, \tau_{1} \leftarrow \tau_{2}, \tau_{1} \rightarrow \tau_{2}, \tau_{1} \leftarrow \tau_{2}$, or $\tau_{1} \stackrel{\tau_{3}}{\rightarrow} \tau_{2}$, is a non-trivial constraint, then $G_{C}$ contains, respectively, an edge $\tau_{1} \rightarrow \tau_{2}($ a $c$-edge $), \tau_{1} \leftarrow \tau_{2}$ (a $p$-edge $), \tau_{1} \rightarrow \tau_{2}$ (a d-edge $)$, $\tau_{1} \leftarrow \tau_{2}$ (an a-edge), or $\tau_{1} \stackrel{\tau_{3}}{\rightarrow} \tau_{2}$ (an s-edge).

Thus, there are five types of edges in $G_{C}: a_{-}, c_{-}, d_{-}$, and $p$ edges (all represented by solid arrows) denote RA, RC, RD, and RP constraints, respectively; and $s$-edges (represented by labelled dashed arrows) denote sibling constraints. We refer to $\tau_{3}$ as the edge label of an $s$-edge $\tau_{1} \stackrel{\tau_{3}}{\rightarrow} \tau_{2}$.

In all the above five edge types, we say that $\tau_{1}$ is the parent of $\tau_{2}$; or equivalently, $\tau_{2}$ is the child of $\tau_{1}$. Note that in the edge specification, $\tau_{1}$ is on the left side of the arrow and $\tau_{2}$ is on the right side of the arrow. Given two nodes $\tau_{1}$ and $\tau_{k}$ in $G_{C}$, we say that $\tau_{1}$ is an ancestor of $\tau_{k}$ (or equivalently, $\tau_{k}$ is a descendant of $\tau_{1}$ ) if there is a sequence of nodes $\tau_{1}, \tau_{2}, \cdots, \tau_{k}$ in $G_{C}$ such that $\tau_{i}$ is the parent of $\tau_{i+1}$ for $i \in[1, k)$. Graphically, each edge in $G_{C}$ is depicted with the parent node shown above the child node.

Consider an edge $e$ with parent node $\tau_{1}$ and child node $\tau_{2}$. If $e$ is an $\ell$-edge, where $\ell \in\{a, c, d, p, s\}$, we say that $\tau_{1}$ is a $\ell$-parent of $\tau_{2}$, and that $\tau_{2}$ is a $\ell$-child of $\tau_{1}$.

To avoid cluttering $G_{C}$, if two nodes in $G_{C}$ are connected by two solid edges (which must necessarily represent one forward and one backward constraint), then these two edges are combined and represented as a single, double-headed solid arrow. For example, if $G_{C}$ contains both edges $\tau_{1} \rightarrow \tau_{2}$ and $\tau_{1} \leftarrow \tau_{2}$, then they can simply be represented by a single edge $\tau_{1} \leftrightarrow \tau_{2}$. In this case, $\tau_{1}$ is a $p$-parent as well as a $c$-parent of $\tau_{2} ; \tau_{2}$ is a $p$-child as well as a $c$-child of $\tau_{1}$

Following [14], we assume that $C$ is available as part of the data, and therefore both closure $(C)$ and $G_{C}$ are computed only once offline. The closure of $C$, closure $(C)$, can be computed in $O\left(|\Sigma|^{2}\right)$. The size of closure $(C)$ is $O\left(|\Sigma|^{2}\right)$. $G_{C}$ consists of $O(|\Sigma|)$ nodes and $O\left(|\Sigma|^{2}\right)$ edges.

Reachable subgraph. Given $\tau_{i} \in V_{C}$ and $L \subseteq \Sigma$, we define $G_{\tau_{i}}^{L}=\left(V_{\tau_{i}}^{L}, E_{\tau_{i}}^{L}\right)$ to be the reachable subgraph of node $\tau_{i}$ in $G_{C}$, where $V_{\tau_{i}}^{L} \subseteq V_{C}$ and $E_{\tau_{i}}^{L} \subseteq E_{C}$ is the set of edges induced by $V_{\tau_{i}}^{L}$

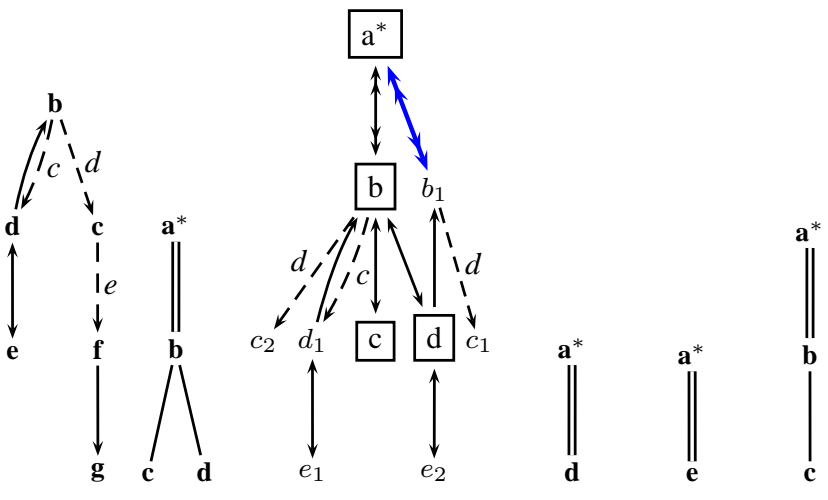
(a) $G_{C}$
(b) Q
(c) $Q_{C}$
(d) $Q_{1}$
(e) $Q_{2}$
(f) $Q_{3}$

in $G_{C} . V_{\tau_{i}}^{L}$ is defined as follows: $\tau_{j} \in V_{\tau_{i}}^{L}$ if one of the following conditions hold:

R1. $\tau_{j}=\tau_{i}$; or

R2. $\tau_{k} \in V_{\tau_{i}}^{L}$ and there is an $a / c / d / p$-edge from $\tau_{k}$ to $\tau_{j}$ in $G_{C}$; or

R3. $\tau_{i} \stackrel{t}{\stackrel{t}{\rightarrow}} \tau_{j}$ is in $G_{C}$ and $t \in L$; or

R4. $\tau_{k} \stackrel{t}{-} \rightarrow \tau_{j}$ is in $G_{C},\left\{\tau_{k}, t\right\} \subseteq V_{\tau_{i}}^{L}$, and $t$ is a $c / p / s$-child of $\tau_{k}$ in $G_{C}$.

Intuitively, a reachable subgraph $G_{\tau_{i}}^{L}$ represents all the nodes in $G_{C}$ that are reachable from node $\tau_{i}$ by traversing the edges in $G_{C} ; L \subseteq$ $\Sigma$ can be used as edge labels in the traversal of s-edges (condition R3).

PCC-pair. For each $s$-edge $\tau_{1} \stackrel{\tau_{2}}{\rightarrow} \tau_{3}$ in $G_{C}$, we refer to the type pair $\left(\tau_{1}, \tau_{2}\right)$ as a parent-conditional-child pair (PCC-pair) with parent type $\tau_{1}$ and conditional child type $\tau_{2}$.

Type \& PCC-pair Equivalence. For each type $\tau_{i} \in V_{C}$, we define $G_{\tau_{i}}^{\emptyset}$ to be the reachable subgraph for $\tau_{i}$; and for each PCC-pair $\left(\tau_{i}, \tau_{j}\right)$ in $G_{C}$, we define $G_{\tau_{i}}^{\left\{\tau_{j}\right\}}$ to be the reachable subgraph for $\left(\tau_{i}, \tau_{j}\right)$.

Let $X$ and $Y$ be a type in $V_{C}$ or a PCC-pair in $G_{C}$. We say that $X$ and $Y$ are equivalent, denoted by $X \equiv Y$, if the reachable subgraphs for $X$ and $Y$ are equal. We use $[X]$ to denote the equivalence class for $X$ (based on type/PCC-pair equivalence); i.e., $[X]=\left\{Y \mid Y\right.$ is a type in $V_{C}$ or a PCC-pair in $\left.G_{C}, Y \equiv X\right\}$.

Given an equivalence class $[X]$, a member $Y \in[X]$ is defined to be minimal if (1) $Y$ is a type; or (2) $Y$ is a PCC-pair $\left(\tau_{i}, \tau_{j}\right)$ such that $\tau_{i} \notin[X]$ and $\tau_{j} \notin[X]$. An equivalence class $[X]$ is a trivial equivalence class if $[X]$ has only one minimal member; otherwise, $[X]$ is a non-trivial equivalence class.

The concept of an equivalence class is very fundamental in our TPQ minimization approach as it is used to characterize important properties of multiple minimal queries in Section 5.

Example 4.1 Consider $\Sigma=\{a, b, c, d, e, f, g\}$ with a set of constraints $C=\{b \stackrel{c}{\rightarrow} d, d, \underset{d}{\rightarrow} c, d \rightarrow e, d \rightarrow e, d \stackrel{f}{\rightarrow} e, d \leftarrow$ $e, b \leftarrow d, b \nleftarrow e, f \rightarrow g, c \stackrel{e}{e} \rightarrow f\}$. The set of non-trivial constraints $C^{\prime}=\{b \stackrel{c}{\rightarrow} d, b \stackrel{d}{\rightarrow} c, d \rightarrow e, d \leftarrow e, b \leftarrow d, f \rightarrow$ 
$g, c \stackrel{e}{\rightarrow} f\}$. The constraint graph $G_{C}$ built from $C^{\prime}$ is shown in Figure 3(a). $G_{C}$ has a $c$-edge (p-edge resp.) from $d$ to $e$ (b resp.); thus $e \in V_{d}^{\emptyset}\left(b \in V_{d}^{\emptyset}\right.$ resp.). Now $b \in V_{d}^{\emptyset}$ has an $s$-child $d \in V_{d}^{\emptyset}$, and $b^{-} \stackrel{d}{\rightarrow} c \in G_{C}$; thus $c \in V_{d}^{\emptyset}$. Therefore, we have $V_{d}^{\emptyset}$ $=\{b, c, d, e\}$. Similarly, $V_{e}^{\emptyset}=V_{b}^{\{c\}}=V_{b}^{\{d\}}=\{b, c, d, e\}$. We have an equivalence class $\{(b, c),(b, d), d, e\}$, of which $(b, c), d$ and $e$ are the only minimal members.

\section{PROPERTIES OF MINIMAL QUERIES}

Previous work on TPQ minimization has shown that for F/FTconstraints, each query has a unique minimal query $[5,14]$. However, beyond these results for F/FT-constraints, there has not been any systematic study and characterization of the properties of minimal queries.

In this section, we characterize important properties of minimal queries under various subclasses of FBST-constraints. First, we present a necessary condition for the existence of multiple minimal queries under FBST-constraints. Then, for each of the constraint classes FBT, FST, and FBST, we characterize the conditions for a query to have multiple minimal queries.

The following result states a necessary condition for the existence of multiple minimal queries under FBST-constraints.

PROPOSITION 5.1. Consider the minimization of a query $Q$ under a set of FBST-constraints $C$. A necessary condition for $Q$ to have multiple minimal queries is the existence of a non-trivial equivalence class in $G_{C}$.

The intuition behind Proposition 5.1 is as follows. Consider two distinct minimal queries $Q_{m}$ and $Q_{m}^{\prime}$ for a query $Q$. Since $Q_{m}$ and $Q_{m}^{\prime}$ are distinct, each minimal query must contain some components that are different from each other. Let $C_{m}$ and $C_{m}^{\prime}$ denote these components of $Q_{m}$ and $Q_{m}^{\prime}$, respectively. However, since $Q_{m}$ and $Q_{m}^{\prime}$ are equivalent, the reachable subgraphs of these components must be the same; i.e., they must both belong to some equivalence class $[X]$ in $G_{C}$. Furthermore, both $C_{m}$ and $C_{m}^{\prime}$ are necessarily minimal members of $[X]$ given that $Q_{m}$ and $Q_{m}^{\prime}$ are minimal queries. Thus, it follows that $[X]$ must be a non-trivial equivalence class in $G_{C}$.

The following result characterizes minimal queries under FBTconstraints.

Proposition 5.2. Let $Q_{m}$ be a minimal query of $Q$ under a set of FBT-constraints $C$. Then $Q$ has another distinct minimal query $Q_{m}^{\prime}$ iff the following two conditions hold:

1. $Q_{m}$ has an ad-edge $(p, x)$, where $x$ is a non-output leaf node; and

2. there exists $\tau_{y} \in[\tau(x)]$ such that
(a) $\tau(x) \leftarrow \tau_{y} \in G_{C}$ and $\tau(p) \not \leq \tau(x)$; or
(b) $\tau_{y} \leftarrow \tau(x) \in G_{C}$ and $\tau(p) \not \leq \tau_{y}$.

Under FBT-constraints, the absence of sibling constraints means that each equivalence class contains only element types.

Based on Proposition 5.2, if $Q_{m}$ is a minimal query of $Q$ under a set of FBT-constraints $C$, then another minimal query of $Q$ can be derived from $Q_{m}$ by changing node $x$ to a $\tau_{y}$-node. Furthermore, all the minimal queries of $Q$ must be of the same size and they differ only in their $a d$-leaf nodes.

Example 5.1 Consider $C=\{c \leftarrow d, c \rightarrow d, a \rightarrow e, e \rightarrow f, e \leftarrow$ $f\}$. We have the two non-trivial equivalence classes in $G_{C}:\{c, d\}$ and $\{e, f\}$. Consider TPQ $Q$ in Figure 4(a) and a minimal query $Q_{1}$ of $Q$ (w.r.t. $C$ ) in Figure 4(b). Since $Q_{1}$ has a non-output adleaf node $c$, and there is a type $d \in[c]$ such that $c \leftarrow d$ and $a \not \leq c$, by Proposition 5.2, $Q$ has multiple minimal queries. Indeed, $Q_{2}$ in Figure 4(c) is another minimal query of $Q$ that is obtained from $Q_{1}$ by changing $c$ to $d$. Note that if $C$ had an additional constraint $a \leq c$, then $Q_{2}$ would not be a minimal query of $Q$. To see this, consider a single data chain $\langle\mathrm{a}\rangle\langle\mathrm{d} /\rangle\langle\mathrm{a} /\rangle$. Clearly, this data (which satisfies $C$ ) is an answer to $Q_{2}$, but not to $Q_{1}$.
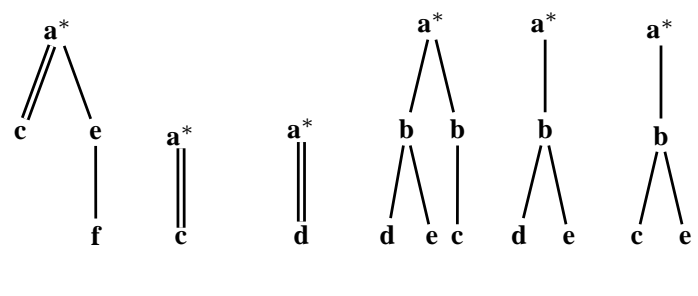
(a) $Q$
(b) $Q_{1}$
(c) $Q_{2}$
(d) $Q^{\prime}$
(e) $Q_{3}$
(f) $Q_{4}$

\section{Figure 4: Illustration of Properties of Minimal Queries}

The following result characterizes minimal queries under FSTconstraints.

PROPOSITION 5.3. Let $Q_{m}$ be a minimal query of $Q$ under a set of FST-constraints $C$. Then $Q$ has another distinct minimal query $Q_{m}^{\prime}$ iff all the following conditions hold:

1. $Q_{m}$ has an pc-edge $(p, x)$, where $x$ is a non-output leaf node; and

2. there exists $\left(\tau(p), \tau_{y}\right) \in[(\tau(p), \tau(x))]$, where $\tau_{y} \in \Sigma$ and $\tau_{y} \neq \tau(x)$.

Under FST-constraints, the absence of backward constraints implies that each equivalence class contains only PCC-pairs.

Based on Proposition 5.3, if $Q_{m}$ is a minimal query of $Q$ under a set of FST-constraints $C$, then another minimal query of $Q$ can be derived from $Q_{m}$ by changing node $x$ to a $\tau_{y}$-node. Furthermore, all the minimal queries for $Q$ must be of the same size and they differ only in their $p c$-leaf nodes.

Example 5.2 Consider $C=\{b \stackrel{c}{\rightarrow} d, b \stackrel{d}{\rightarrow} d, c\}$. We have $(b, c) \equiv$ $(b, d)$. TPQ $Q_{3}$ (Figure 4(e)) is a minimal query of TPQ $Q^{\prime}$ (Figure $4(\mathrm{~d})$ ). Since $Q_{3}$ has a non-output $p c$-leaf $d$, and $G_{C}$ has an equivalence class $[(b, d)]$ where $(b, c) \in[(b, d)]$, by Proposition 5.3, $Q^{\prime}$ has another minimal query $Q_{4}$ (Figure 4(f)), which is obtained from $Q_{3}$ by changing $d$ to $c$.

Finally, the following result characterizes minimal queries under FBST-constraints.

PROPOSITION 5.4. Let $Q_{m}$ be a minimal query of $Q$ under a set of FBST-constraints $C$. Then $Q$ has another distinct minimal query $Q_{m}^{\prime}$ iff one of the following conditions hold:

1. $Q_{m}$ satisfies conditions 1 and 2 of Proposition 5.2;

2. $Q_{m}$ satisfies conditions 1 and 2 of Proposition 5.3;

3. $Q_{m}$ satisfies all of the following conditions:

(a) $Q_{m}$ has an ad-edge $(p, x)$, where $x$ is a non-output leaf node; and 


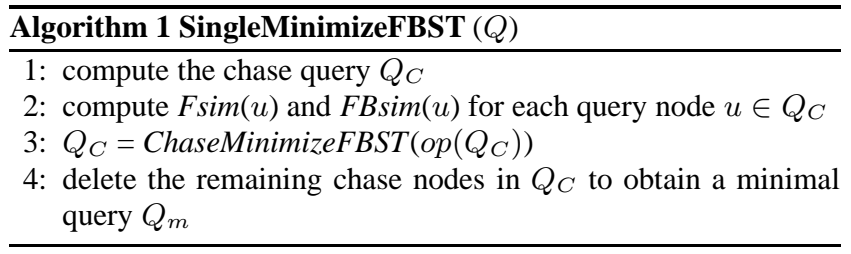

(b) there exists a minimal member $\left(\tau_{y}, \tau_{z}\right) \in[\tau(x)]$, where $\tau_{y} \in \Sigma$ and $\tau_{z} \in \Sigma$.

4. $Q_{m}$ satisfies all of the following conditions:

(a) $Q_{m}$ has an pc-edge $(p, x)$, where $x$ is a non-output leaf node;

(b) $p$ is an ad-child; and

(c) there exists some element type $\tau_{i} \in[(\tau(p), \tau(x))]$.

Under FBST-constraints, an equivalence class may contain both single types as well as PCC-pairs.

Based on Proposition 5.4, if $Q_{m}$ is a minimal query of $Q$ under a set of FBST-constraints $C$, then another minimal query $Q_{m}^{\prime}$ of $Q$ can be derived from $Q_{m}$ as follows. If $Q_{m}$ satisfies condition 1 or 2 , then $Q_{m}^{\prime}$ can be derived from $Q_{m}$ by changing node $x$ to a $\tau_{y}$-node. If $Q_{m}$ satisfies condition 3 , then $Q_{m}^{\prime}$ can be derived from $Q_{m}$ by changing node $x$ to a $p c$-edge $\left(\tau_{y}, \tau_{z}\right)$. Finally, if $Q_{m}$ satisfies condition 4 , then $Q_{m}^{\prime}$ can be derived from $Q_{m}$ by changing the $p c$-edge $(p, x)$ to a $\tau_{i}$-node. Furthermore, if $Q$ has multiple minimal queries, their sizes could differ.

Example 5.3 (continued from Example 4.1) TPQ $Q_{1}$ (Figure 3(d)) is a minimal query of TPQ $Q$ (Figure 3(b)). From Example 4.1, we have $[d]=\{d, e,(b, d),(b, c)\}$. By the condition set 1 of Proposition 5.4 ( $Q_{1}$ has a non-output $a d$-leaf $d ; e \in[d]$ and $d \leftarrow e$ ), $Q$ has distinct minimal queries. By changing ad-leaf $d \in Q_{1}$ to minimal members of $[d]$, $e$ or $(b, c)$, we have two other minimal queries $Q_{2}$ (Figure 3(e)) and $Q_{3}$ (Figure 3(f)).

\section{MINIMIZE WITH FBST-CONSTRAINTS}

In this section, we present algorithms to minimize TPQs under the broadest class of FBST-constraints. We first present techniques for computing a single minimal query (Sections 6.1 to 6.4), and then extend the approach to enumerate all minimal queries (Section 6.5).

Our overall approach to compute a single minimal query for a TPQ $Q$ (w.r.t. a set of constraints $C$ ) is shown in Algorithm 1 and consists of four main steps. The first step is to compute the chase query $Q_{C}$ of the input query; the goal is to integrate the relevant constraints from $C$ into $Q$ to created an augmented query $Q_{C}$ that contains additional chase nodes. The details of the chase query computation are discussed in Sections 6.1 and 6.3. The second step (Section 6.2) is to compute simulation relations for the nodes in $Q_{C}$; the purpose of this step is to enable the detection of redundant nodes in $Q$ to generate a minimal query of $Q$, which is performed in the third step (Section 6.4). Finally, the fourth step simply removes any remaining chase nodes from $Q_{C}$ to obtain a minimal query of $Q$.

While our overall approach follows the same principle as the previous work for query minimization with FT-constraints [14], the TPQ minimization problem with FBST-constraints is a more challenging task due to the intricacies of dealing with BS-constraints which requires the development of several new techniques:
1. The computation of closure $(C)$ requires new inference rules to handle BS-constraints (R9-R21 presented in Section 4.1).

2. When building $Q_{C}$, the presence of B-constraints requires a more intricate augmented chase computation (i.e., the $a d-$ edge augmentation in Section 6.3) to address the inadequacy of the conventional chase computation (Section 6.1). Moreover, the detection of redundant nodes now requires computing forward \& backward simulation which is more involved rather than computing forward simulation.

3. The presence of S-constraints, which represent conditional constraints, also demands some subtle extensions to the chase and minimization process (Sections 6.1 and 6.4).

4. The presence of BS-constraints leads to some fundamental new properties of minimal queries identified in Section 5; specifically, the possibility of multiple minimal queries with different sizes requires new techniques that exploit properties of minimal queries to efficiently enumerate all minimal queries (Section 6.5).

\subsection{The Chase Procedure}

Given a TPQ $Q$, the chase query of $Q$, denoted by $Q_{C}$, is computed using a two-step procedure:

S1. Initialize $Q_{C}$ to be $Q$. For every query node $u$ in $Q_{C}$, attach the reachable subgraph $G_{\tau(u)}^{L}$ to $u$, where $L=\{\tau(v) \mid \mathrm{v}$ is a $p c$-child of $\mathrm{u}$ in $Q\}$.

S2. Augment $Q_{C}$ with additional $a d$-edges.

Step S1 initializes $Q_{C}$ to be $Q$, and then enhances each node $u$ in $Q$ with additional nodes from its reachable subgraph based on $\tau(u)$ and the types of $u$ 's child nodes in $Q$. The nodes and edges in $Q_{C}$ can be classified into two types: the nodes (edges) that are originally in $Q$ are called original nodes (edges) and the attached nodes (edges) added to $Q_{C}$ are called chase nodes (edges).

Step S2, which is referred to as the augment chase step, then inserts into $Q_{C}$ a set (possibly empty) of additional $a d$-edges referred to as augmented edges. This important augmentation step is necessary due to the presence of backward constraints. The need for the augment chase step will be illustrated in Example 6.1; however, we will defer a detailed discussion of this step to Section 6.3 after we have explained the identification of redundant nodes in $Q_{C}$ using FBsimulation in Section 6.2.

Graphically, we distinguish between original and chase nodes in $Q_{C}$ by showing the former as boxed nodes and the latter as unboxed nodes. For ease of identification of nodes of the same type in $Q_{C}$, we also add subscripts to the node labels when convenient. For consistency of edge notations, the non-arrowed original edges in $Q$ are represented using arrowed edges in $Q_{C}$ as follows. Let $e=(x, y)$ be an original edge in $Q$ with parent node $x$ and child node $y$. Then $e$ is represented in $Q_{C}$ as $x \leftrightarrow y$ if $e$ is a $p c$-edge; otherwise, $e$ is an $a d$-edge and it is represented as $\mathrm{x} \leftrightarrow \mathrm{y}$. Finally, to distinguish normal $a d$-edges from augmented $a d$-edges (introduced by step S2), the latter are shown as bold edges.

Note that the conventions and definitions introduced in Section 4 for $G_{C}$ also apply to $Q_{C}$.

The following example illustrates step S1 of the chase computation and motivates the need for the augment chase step S2.

Example 6.1 Consider $Q$ in Figure 5 with constraints $C=\{c \nleftarrow$ $e, e \nVdash a\}$. We explain how step S1 of the chase is performed on $Q$ to derive $Q_{C}$ (shown in Figure 5(b)). The reachable subgraph $G_{a}^{\emptyset}$ is attached to nodes $a^{*}$, i.e., two chase nodes $e_{1}$ and $c_{1}$. The 
reachable subgraph $G_{e}^{\emptyset}$ is attached to nodes $e$, i.e., chase node $c_{2}$. Specifically, no chase nodes are attached to the remaining nodes of $Q$ since their reachable graphs contain only the node itself.

Note that there is an augmented edge between $e_{1}$ and $b_{1}$ in $Q_{C}$; this is added by the augment chase step $\mathrm{S} 2$. To appreciate why this addition is necessary, assume for the moment that the augmented edge is not present in $Q_{C}$. Observe that the output node $a$ has two ancestor paths of nodes: one leads to $c$ while the other leads to $c_{1}$. The purpose of adding the augmented edge $\left(e_{1}, b_{1}\right)$ is to explicitly connect these two related paths to enable a correct detection of redundant nodes using FBsimulation (to be discussed in the next section). Specifically, the original nodes $c, e$, and $b_{2}$ are all actually redundant and need to be removed to generate a correct minimal query $Q_{2}$. However, without the augmented edge $\left(e_{1}, b_{1}\right)$, the FBsimulation technique would wrongly identify these three nodes as non-redundant.

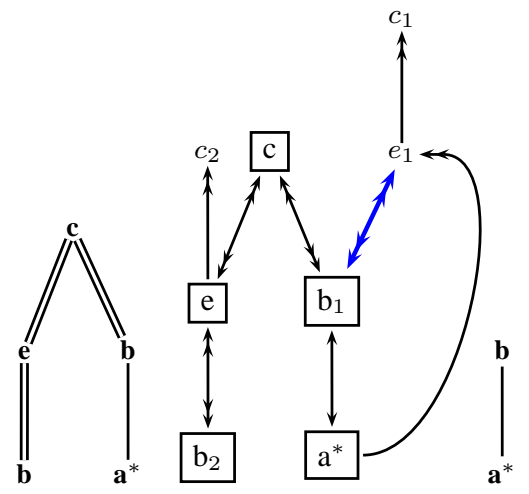

(a) $Q$

(b) $Q_{C}$

(c) $Q_{2}$

\section{Figure 5: Example of the Chase Procedure}

Complexity of the chase. For a node $u$ in $Q$, the reachable subgraph $G_{\tau(u)}^{L}$ is added to $u$ in step S1. Since $G_{\tau(u)}^{L}$ can be as large as $G_{C}$, it consists of $O(|\Sigma|)$ nodes and $O\left(|\Sigma|^{2}\right)$ edges; thus the resultant graph of step $\mathrm{S} 1$ comprises of $O(n|\Sigma|)$ nodes and $O\left(n|\Sigma|^{2}\right)$ edges. The $a d$-edge augmentation in step S2 adds in $O\left(n^{2}|\Sigma|\right)$ edges. Thus the chase of $Q, Q_{C}$, comprises of $O(n|\Sigma|)$ nodes and $O\left(n^{2}|\Sigma|+n|\Sigma|^{2}\right)$ edges.

For each node $u, G_{\tau(u)}^{L}$ can be computed by at most one traversal on $G_{C}$, which takes $O\left(|\Sigma|^{2}\right)$ time. Hence step S1 can be done in $O\left(n|\Sigma|^{2}\right)$ time. Step S2 takes $O\left(n^{3}|\Sigma|\right)$ time. The overall time complexity of the chase procedure is $O\left(n^{3}|\Sigma|+n|\Sigma|^{2}\right)$.

\subsection{Forward \& Backward Simulation}

Once the chase query $Q_{C}$ has been computed, any redundant nodes in $Q_{C}$ can be detected and eliminated based on the concept of forward-backward simulation (FBsimulation) [4].

The FBsimulation on the nodes of $Q_{C}$ is the largest binary relation $\preceq_{F B}$ on the nodes of $Q_{C}$ such that $u \preceq_{F B} v$ iff all the following conditions hold:

(1) Preserve node types: $\tau(u) \leq \tau(v)$; moreover, if $u=o p(Q)$, then $v=o p(Q)$;

(2) Preserve parent-relationships: if $u$ has a $p$-parent $u^{\prime}$, then $v$ has a $p$-parent or $c$-parent or $s$-parent $v^{\prime}$ s.t. $u^{\prime} \preceq_{F B} v^{\prime}$;
(3) Preserve child-relationships: if $u$ has a $c$-child $u^{\prime}$, then $v$ has a $p$-child or $c$-child or $s$-child $v^{\prime}$ s.t. $u^{\prime} \preceq_{F B} v^{\prime}$;

(4) Preserve ancestor-relationships: if $u$ has an $a$-parent $u^{\prime}$, then $v$ has an ancestor $v^{\prime}$ s.t. $u^{\prime} \preceq_{F B} v^{\prime}$;

(5) Preserve descendant-relationships: if $u$ has a $d$-child $u^{\prime}$, then $v$ has a descendant $v^{\prime}$ s.t. $u^{\prime} \preceq_{F B} v^{\prime}$.

The forward simulation $\preceq_{F}$ on the nodes of $Q_{C}$ is computed using only conditions (1), (3), and (5); while the backward simulation $\preceq_{B}$ on the nodes of $Q_{C}$ is computed using only conditions (1), (2), and (4).

If $u \preceq_{F} v\left(u \preceq_{B} v, u \preceq_{F B} v\right.$ resp.), we say that $v$ is a forward (backward, forward-backward resp.) simulator of $u$. Given a node $u$ in $Q_{C}$, we use $F \operatorname{sim}(u), B \operatorname{sim}(u)$, and $F B \operatorname{sim}(u)$ to refer to the set of forward, backward, and forward-backward simulators of $u$, respectively. Note that $F B \operatorname{sim}(u)$ is always a subset of $F \operatorname{sim}(u)$ and $\operatorname{Bsim}(u)$.

Algorithms for computing forward simulation Fsim( $u$ in TPQs were presented in [14]. These algorithms could be extended to compute $F B$ sim relation on $Q_{C}$ for our context. Such modified algorithms take $O\left(n^{3}|\Sigma|+n^{2}|\Sigma|^{2}\right)$ time to compute $\operatorname{Fsim}(u)$ and $\operatorname{FB} \operatorname{sim}(u)$ for every original node $u$ in $Q_{C}$.

Example 6.2 (continued from Example 4.1) For $Q_{C}$ in Figure 3, we have $F \operatorname{sim}(a)=F B \operatorname{sim}(a)=\{a\}, F \operatorname{sim}(b)=F B \operatorname{sim}(b)=$ $\left\{b, b_{1}\right\}, F \operatorname{sim}(c)=F B \operatorname{sim}(c)=\left\{c, c_{1}, c_{2}\right\}$, and $F \operatorname{sim}(d)=$ $F B \operatorname{sim}(d)=\left\{d, d_{1}\right\}$.

We can now identify redundant nodes in $Q_{C}$ based on the following result.

LEMMA 6.1. Let $u \in Q_{C}$ be a non-redundant original node.

(1) An original p-parent $p$ of $u$ is redundant iff $u$ has another p-parent $p^{\prime} \in F B \operatorname{sim}(p)$;

(2) An original a-parent $p$ of $u$ is redundant iff $u$ has another ancestor $p^{\prime} \in F B \operatorname{sim}(p)$;

(3) An original c-child $c$ of $u$ is redundant iff $u$ has another $c$ child or s-child $c^{\prime} \in F \operatorname{sim}(c)$;

(4) An original d-child $c$ of $u$ is redundant iff $u$ has another descendant $c^{\prime} \in F \operatorname{sim}(c)$.

Lemma 6.1 is extension of a result in [14], where the focus there was identifying redundant $c$-child and $d$-child nodes using only forward simulation; i.e. conditions (3) and (4). The new two conditions (1) and (2) for identifying redundant $p$-parent and $a$-parent nodes, however, require a stronger test based on FBsimulation. We illustrate the necessity of FBsimulation using the following example.

Example 6.3 Consider $Q$ in Figure 6(a) with $C=\{a \leftarrow b, b \rightarrow$ $d, b \leftarrow c\} . G_{C}$ and $Q_{C}$ are shown in Figures 6(b)\&(c). Note that the original node $b$ is not redundant. In $Q_{C}$, we have $b_{1} \notin$ $F B \operatorname{sim}(b)$, which is consistent with the fact that $b$ is not redundant. Thus, node $b$ remains in $Q$ 's minimal query $Q_{m}$ shown in Figure 6(d). Observe that node $c$ has another $p$-parent $b_{1}$ in $Q_{C}$ such that $b_{1} \in F \operatorname{sim}(b)$ and $b_{1} \in B \operatorname{sim}(b)$. However, it is incorrect to conclude from $b_{1} \in F \operatorname{sim}(b)$ and $b_{1} \in B \operatorname{sim}(b)$ that $b$ is redundant. 


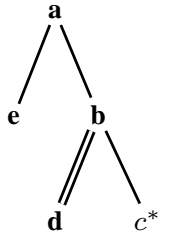

(a) $Q$

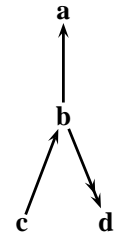

(b) $G_{C}$

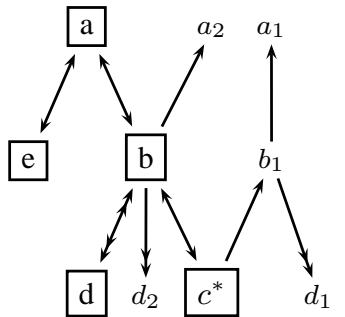

(c) $Q_{C}$

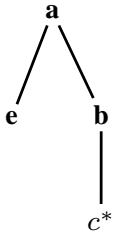

(d) $Q_{m}$

\section{Figure 6: FBsimulation in Checking Redundant Parent}

\subsection{Augmented Chase}

We are now ready to explain the details of the important augment chase step (step S2 in Section 6.1). Due to the presence of backward constraints, a node in $Q_{C}$ could have multiple path of ancestor nodes which are necessarily related since each node in a query tree cannot have multiple unrelated paths of ancestor nodes. Thus, the augment chase step S2 is necessary to check for possible implied relationships among multiple ancestor paths. Without this important step, redundant nodes might not be correctly detected leading to incorrect minimal queries.

Recall that Example 6.1 illustrated the need to perform $a d$-edge augmentation between an original node and a chase node in $Q_{C}$. In general, $a d$-edge augmentation is necessary for any pair of related ancestor nodes. In the next example, we illustrate another scenario where $a d$-edge augmentation is required between a pair of related chase nodes.

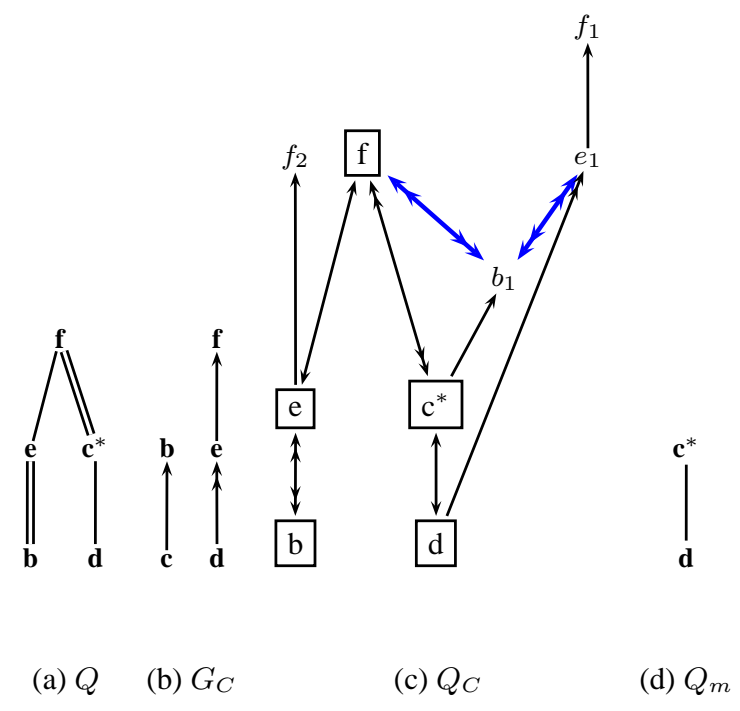

Figure 7: Example of $a d$-edge Augmentation

Example 6.4 Consider $Q$ in Figure 7(a) with $C=\{b \leftarrow c, e \nleftarrow$ $d, f \leftarrow e\} . G_{C}$ and $Q_{C}$ are shown in Figures 7(b) and (c), respectively. Note that $Q_{C}$ has an augmented $a d$-edge between the chase nodes $b_{1}$ and $e_{1}$ due to the tree structure property $\left(b_{1}\right.$ is a $p$-parent of $c, c$ is a $p$-parent of $d$, and $e_{1}$ is an $a$-parent of $d$ ). Similarly there is an augmented $a d$-edge between $b_{1}$ and $f$. From $Q_{C}$, we can identify that the original nodes $f, e$, and $b$ are redundant (due to their forward-backward simulators $f_{1}, e_{1}$, and $b_{1}$, respectively), leading to the minimal query $Q_{m}$ in Figure 7(d). Without the augmented $a d$-edges, $Q_{m}$ cannot be derived based on FBsimulation.

An important point to emphasize is that it is only necessary to perform edge augmentation for $a d$-edges: adding $p c$-edges between related ancestor nodes is not sound. We illustrate the intuition behind this with the following example.

Example 6.5 Consider $Q_{1}$ in Figure 8 with $C=\{b \leftarrow c\}$. If $p c$-edge augmentation is done, then the resultant chase query $Q_{C}^{\prime}$ is shown in Figure 8 with an augmented $p c$-edge between $a$ and $b_{1}$. We then have $b_{1} \in F B \operatorname{sim}(b)$, leading to a wrong conclusion that $b$ is redundant and an incorrect minimization of $Q_{1}$ to $Q_{2}$ that results from the removal of $b$ from $Q^{\prime}$. To see that $Q_{1}$ and $Q_{2}$ are not equivalent, consider data $\langle a\rangle\langle d\rangle\langle b\rangle\langle c /\rangle\langle b /\rangle\langle d /\rangle\langle a /\rangle$. $Q_{2}$ matches the data, while $Q_{1}$ does not.

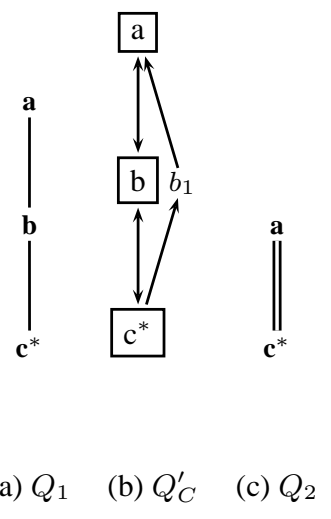

Figure 8: Example of $a d$-edge Augmentation

\subsubsection{Augmentation Algorithm}

This section presents the algorithm to perform the augment chase step S2. The key challenge of this step is: given a node $u$ in $Q_{C}$ which has two ancestor paths of nodes, how to characterize the pair(s) of related nodes along these paths that need to be connected with augmented edges. We shall formalize this using the concepts of ancestor path, $p$-path and compatible nodes.

A path of nodes $\left(w_{1}, \cdots, w_{n}\right)$ in $Q_{C}, n \geq 1$, is said to be an ancestor path of $w_{1}$ if (1) $w_{i}$ is a $p / a$-parent of $w_{i-1}$ for each $i \in(1, n]$; and (2) $w_{n}$ has no $p / a$-parent node in $Q_{C}$. A path of nodes $\left(v_{1}, \cdots, v_{m}\right)$ in $Q_{C}, m \geq 1$, is said to be a $p$-path of $v_{1}$ if (1) $v_{i}$ is a $p$-parent of $v_{i-1}$ for each $i \in(1, m]$; and (2) $v_{m}$ has no $p$-parent node in $Q_{C}$.

A node $v_{i}$ is said to be compatible with another node $w_{i}$ iff $\tau\left(v_{i}\right) \leq \tau\left(w_{i}\right)$ or $\tau\left(w_{i}\right) \leq \tau\left(v_{i}\right)$.

Consider a node $u$ in $Q_{C}$ that has both a $p$-path $p_{v}=\left(u, v_{1}, \cdots\right.$, $\left.v_{j}, \cdots, v_{k}, \cdots, v_{m}\right)$ as well as another ancestor path $p_{w}=$ $\left(u, w_{1}, \cdots, w_{j}, \cdots, w_{n}\right)$, where $1 \leq j \leq k \leq m$ and $j \leq n$. Figure 9 illustrates the situations where an augmented edge is required to connect a pair of related nodes in $p_{v}$ and $p_{w}$, which are characterized as follows. An ad-edge needs to be augmented for the following two cases.

Case 1. An $a d$-edge is added between $w_{k+1}$ (the ancestor) and $v_{k}$ (the descendant), if the following two conditions hold:

1. $v_{i}$ is compatible with $w_{i}$ for each $i \in[1, k]$; and

2. $w_{k+1}$ is an $a$-parent of $w_{k}$. 
Case 2. An $a d$-edge is added between $w_{j}$ (the ancestor) and $v_{k}$ (the descendant), if all the three conditions hold:

1. $v_{i}$ is compatible with $w_{i}$ for each $i \in[1, j-1]$; and

2. $w_{j}$ is an $a$-parent of $w_{j-1}$; and

3. (a) $v_{k}$ is the last node in $p_{v}$ (i.e., $k=m$ ), and $v_{s}$ is not compatible with $w_{j}$ for each $s \in[j, k]$; or

(b) $w_{j}$ is not compatible with $v_{t}$ for each $t \in[j, k]$, and $v_{k+1}$ is compatible with $w_{j}$ where $k \in[j, m-1]$.

Figure 9(a) shows Case 1, where $p_{v}$ and $p_{w}$ have pairwise compatible nodes $w_{i}$ and $v_{i}$ for $i \in[1, k]$, and $w_{k+1}$ is an $a$-parent of $w_{k}$. The tree-structure property requires $w_{k+1}$ to be an ancestor of $v_{k}$. Figure 9(b) shows Case 2 where condition 3(a) holds. Here, $p_{v}$ and $p_{w}$ have pairwise compatible nodes $w_{i}$ and $v_{i}$ for $i \in[1, j)$. Node $w_{j-1}$ has an $a$-parent $w_{j}$, and $v_{s-1}$ has a $p$-parent $v_{s}$ which is not compatible with $w_{j}$ for $s \in[j, k]$. Clearly, node $v_{k}$ must be a descendant of $w_{j}$ based on the tree structure property. Note that if $w_{j}$ and $v_{k}$ were compatible, then adding the $a d$-edge $\left(w_{j}, v_{k}\right)$ would be incorrect as $w_{j}$ and $v_{k}$ might be the same node instead of being an ancestor-descendant pair of nodes. The reasoning for Case 2 where condition 3(b) holds (depicted in Figure 9(c)) is similar.

Note that for all such augmented $a d$-edges, the implied descendant always belongs to the $p$-path. The nodes in Figure 9 can be chase or original nodes. The edge $\left(w_{1}, u\right)$ can be either a $p c$-edge or an $a d$-edge.
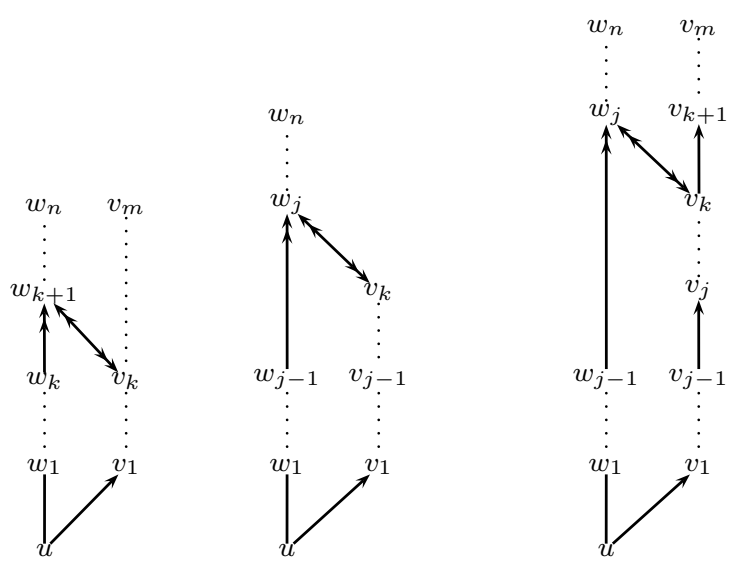

(a) Case 1

(b) Case 2 Condition 3(a)

(c) Case 2 Condition 3(b)

\section{Figure 9: Illustration of Augmentation Algrithm}

We propose an algorithm, ad-augmentation (Algorithm 2), to augment all the necessary $a d$-edges between each $p$-path and ancestor path of an original node $u$, which essentially follows the above fundamental principle.

Let $Q_{C}^{u}$ be the subgraph of $Q_{C}$ consisting of $u$, and the ancestor nodes of $u$ that are also chase nodes of $u$. Let $Q_{C}^{u+}$ be the subgraph of $Q_{C}$ consisting of $u$ and all ancestor nodes of $u$, which can be original or chase nodes. Thus, $Q_{C}^{u}$ is a subgraph of $Q_{C}^{u+}$. Each original query node has exactly one $p$-path $l_{c}$ in $Q_{C}^{u}$, and one or more $p$-paths $l_{i}$ in $Q_{C}^{u}$. We have noted that all the ancestor paths of $u$ are contained in $Q_{C}^{u+}$, and that every $p$-path $l_{i}$ of $u\left(l_{i} \neq l_{c}\right)$ is necessarily a $p$-path of $u$ 's original $p$-parent. Instead of directly applying the above principle to every combination of a $p$-path and

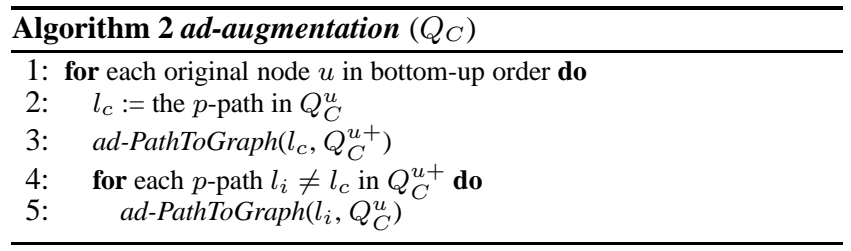

an ancestor path of $u$, we apply the principle to (1) $l_{c}$ with $Q_{C}^{u+}$ (line 3 of Algorithm 2), and (2) $l_{i}$ with $Q_{C}^{u}$ for each $l_{i} \neq l_{c}$ (lines 4-5 of Algorithm 2).

The function ad-PathToGraph used in Algorithm 2 augments the necessary $a d$-edges from the nodes in a $p$-path of $u, l_{p}$, to the nodes in the subgraph $Q_{s}$ (either $Q_{C}^{u}$ or $Q_{C}^{u+}$ ) rooted at $u$, which closely follows the above fundamental principle. Function ad-PathToGraph takes $O\left(n\left|V_{s}\right|\right)$ time and adds at most $O\left(\left|V_{s}\right|\right)$ $a d$-edges, where $V_{s}$ is the set of nodes in $Q_{s}$.

Example 6.6 Consider $Q$ in Figure 3 with the same constraints as in Example 4.1. $Q_{C}$ is shown in Figure 3(c). The chase nodes in $Q_{C}$ is added during step S1 of the chase by attaching the reachable subgraphs. Note that the chase edge between $a$ and $b_{1}$ is added during step S2 of the chase.

Complexity of ad-augmentation $Q_{C}^{u+}$ (may be as big as $Q_{C}$ ) has $O(n|\Sigma|)$ nodes, and $Q_{C}^{u}$ (may be as big as $G_{C}$ ) has $O(|\Sigma|)$ nodes. The number of $p$-paths of $u$ in $Q_{C}$ is bounded by $O(n)$. Algorithm ad-augmentation takes $=O\left(n^{3}|\Sigma|\right)$ time, and adds $O\left(n^{2}|\Sigma|\right)$ $a d$-edges.

\subsection{Generating a Minimal Query}

In this section, we present the algorithm ChaseMinimi zeFBST (shown in Algorithm 3) to compute a single minimal query of an input query $Q$ w.r.t. a set of FBST-constraints.

The input to ChaseMinimizeFBST is a non-redundant original node $u$ in $Q_{C}$, and all redundant original nodes in $Q_{C}$ are detected (by applying Lemma 6.1) and removed via recursive calls to ChaseMinimizeFBST. Each query $Q$ has at least one nonredundant node given by its output node $o p(Q), u=o p(Q)$ in the first call to ChaseMinimizeFBST. Steps 2 to 6 check whether the $p$-parent and $a$-parent $v$ of $u$ in $Q_{C}$ is redundant. If $v$ is redundant, $v$ as well as its chase nodes (if any) are removed. If $v$ is not redundant, a recursive call to ChaseMinimi zeFBST is made with $v$ as the input parameter. Similarly, steps 7 to 13 check and remove any redundant $c$-child and $d$-child nodes of $u$. Nodes that have been checked are marked to avoid repeated redundant checkings.

It is important to point out that due to possible sibling constraints in $C$, the removal of redundant $c$-child nodes in $Q_{C}$ entails some additional checking (in steps 10 and 11) for correctness. Specifically, if an original $c$-child $v$ of $u$ is detected to be redundant, it is necessary to also check whether $u$ has another $c$-child of type $\tau(v)$. If there is no such $c$-child, then $s$-child nodes of $u$ with edge label $\tau(v)$ must also be deleted. This is due to the fact that the condition ( $u$ has a $c$-child of type $\tau(v)$ ) no longer exists. We illustrate this subtle point using the following example.

Example 6.7 Consider $Q$ in Figure 10(a) with $G_{C}$ shown in Figure $10(\mathrm{~b}) . Q_{C}$ is shown in Figure 10(c). ChaseMinimizeFBST identifies the original $c$-child $b$ of node $a$ to be redundant (because $a$ has another $c$-child $\left.b_{1} \in F B \operatorname{sim}(b)\right)$ and $b$ is deleted. In addition, due to steps 10 and 11, ChaseMinimizeFBST also finds that $a$ has no other $c$-child of type $b$. Consequently, the $c$-child $c_{1}$ with edge label $b$ is deleted together with $b$, leading to a mini- 


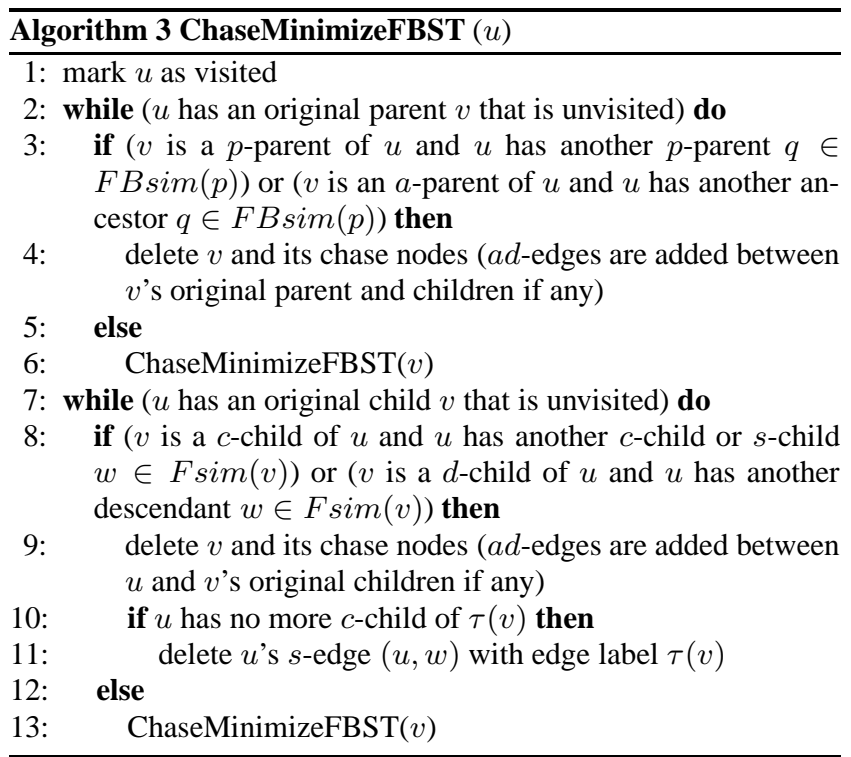

mal query $Q_{2}$ (Figure 10(e)). Note that had the chase node $c_{1}$ not been deleted together with $b, a$ 's original $c$-child $c$ would have been wrongly detected to be redundant (due to the fact that $a$ has another $c$-child $c_{1} \in F B \operatorname{sim}(c)$ ), leading to an incorrect result.

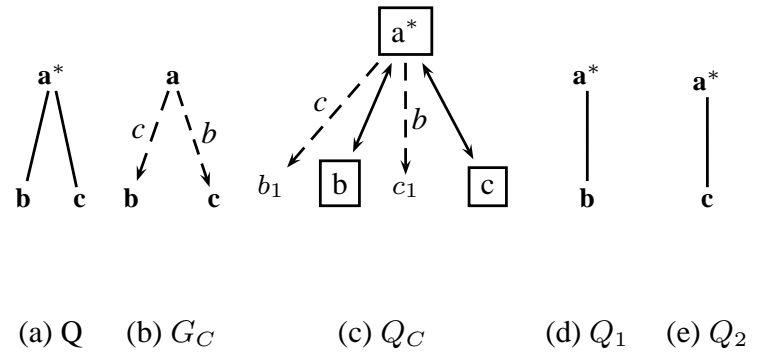

Figure 10: Lines 10 and 11 of ChaseMinimizeFBST

Example 6.8 (continued from example 6.2) For $Q_{C}$ in Figure 3(c), starting from output node $a$, SingleMinimizeFBST finds the $d$-child $b$ of $a$ is redundant as $a^{*}$ has another descendant node $b_{1} \in F B \operatorname{sim}(b)$. Then $b$ and $b$ 's chase nodes $\left(c_{2}, d_{1}\right.$ and $\left.e_{1}\right)$ are deleted. Child nodes $c$ and $d$ of $b$ are connected to $a$ using $a d$-edges. Now $c$ is a $d$-child of $a$, and Lemma 6.1 finds it redundant as $a$ has another descendant $b_{1} \in F B \operatorname{sim}(b)$. Node $c$ is then deleted. SingleMinimizeFBST outputs a minimal query $Q_{1}$ (Figure 3(d)) after deleting the remaining chase nodes.

Complexity of ChaseMinimizeFBST The worst case of algorithm ChaseMinimizeFBST occurs when each redundancy check of Lemma 6.1 enables only one node removal (i.e., one node is deleted at a time). For a non-redundant node $p$, for each child (parent resp.) $u$ of $p$, it takes time proportional to the number of descendant (ancestor resp.) nodes of $p$ to check whether $u$ is redundant. Hence the time of checking whether $u$ is redundant using Lemma 6.1 is $O\left(\left|V_{C}\right|\right)=O(n|\Sigma|)$ where $V_{C}$ is the set of nodes in $Q_{C}$. Deleting $u$ and its chase nodes takes $O(n|\Sigma|)$ time. Thus the worst case of algorithm ChaseMinimi zeFBST takes $O(n *(n|\Sigma|))=O\left(n^{2}|\Sigma|\right)$ time.

For algorithm SingleMinimizeFBST, we have Lemma 6.2 .
LEMMA 6.2. Algorithm SingleMinimizeFBST correctly computes a minimal query of a given TPQ $Q$ in the presence of a set of FBST-constraints. The time-complexity of SingleMinimizeFBST is $O\left(n^{3}|\Sigma|+n^{2}|\Sigma|^{2}\right)$.

\subsection{Enumerating All Minimal Queries}

In this section, we present an algorithm (referred to as MultipleMinimizeFBST) to enumerate all the minimal queries of an input query $Q$ w.r.t. a set of FBST-constraints. The algorithm consists of two key steps. First, SingleMinimizeFBST is used to generate a single minimal query $Q_{m}$ of $Q$. Next, by applying Proposition 5.4, the remaining minimal queries of $Q$ (if any) can be generated by modifying $Q_{m}$. Specifically, additional minimal queries of $Q$ are enumerated from $Q_{m}$ by replacing each $X$, where $X$ is an $a d$-leaf node or a parent-child edge in $Q_{m}$ that satisfies the conditions of Proposition 5.4, with the minimal members in the equivalence class $[X]$.

The time complexity of MultipleMinimizeFBST is $O\left(\max \left\{n^{3}|\Sigma|+n^{2}|\Sigma|^{2},\left|Q_{M}\right|\right\}\right)$, where $O\left(n^{3}|\Sigma|+n^{2}|\Sigma|^{2}\right)$ is the time complexity of algorithm SingleMinimizeFBST; and $Q_{M}$ is the set of distinct minimal queries of $Q$, which could be exponential in the input query size.

Example 6.9 (continued from Example 6.8) From Example 6.8, we know that $Q_{1}$ (Figure 3(d)) is a minimal query of $Q$ (Figure 3(b)). $Q_{1}$ has one $a d$-leaf node, $d$ and no parent-child edges $(u, v)$ where $v$ is a leaf. Following Proposition 5.4, $Q_{1}$ has two other minimal queries, as shown in Figures 3(e) and (f).

\section{MINIMIZE WITH FST-CONSTRAINTS}

In this section, we consider the TPQ minimization problem under FST-constraints. Our key result here is that in the absence of backward constraints, a query can be minimized without explicitly computing the chase of the query, resulting in a more efficient approach. Section 7.1 first presents a new algorithm for FTconstraints. Our algorithm has a time complexity of $O\left(n|\Sigma|+n^{2}\right)$, which improves over the $O\left(n^{4}\right)$ time-complexity of the state-ofthe-art algorithm [14]. In Section 7.2, we extend our approach to handle FST-constraints.

For convenience, we use simulate to mean forward simulate and $\operatorname{sim}(u)$ to mean $F \operatorname{sim}(u)$ in this section.

Let $V$ denote the set of nodes in a query $Q$, and let $S \subseteq V$ be a subset of query nodes, and let $T \subseteq \Sigma$ be a subset of element types. We define type $(S)=\{\tau(v) \mid v \in S\}$, node $(T)=\{v \in$ $V \mid \tau(v) \in T\}, \operatorname{par}(S)=\{v \in V \mid v$ has a $p c$-child in $S\}$, and $\operatorname{anc}(S)=\{v \in V \mid v$ has a descendant in $S\}$.

\subsection{Minimize with FT-constraints}

Our new improved algorithm is based on the idea of type simulators. The complexity reduction of our algorithm is essentially achieved by avoiding the construction of the chase query $Q_{C}$ and the subsequent computation of simulation relation on $Q_{C}$, both of which are costly.

Type simulators. Consider a query node $u$ and a type $t \in \Sigma$. Formally, we say that $t$ simulates $u$ (denoted by $u \preceq t$ ) if all the following conditions hold:

1. $u$ is neither the query output node nor its ancestor;

2. $t \leq \tau(u) \in$ closure $(\mathrm{C})$;

3. for each $p c$-child $v$ of $u$, there exists a type $t^{\prime} \in \Sigma$ such that $t \rightarrow t^{\prime} \in \operatorname{closure}(\mathrm{C})$ and $v \preceq t^{\prime}$; 
4. for each $a d$-child $v$ of $u$, there exists a type $t^{\prime} \in \Sigma$ such that $t \rightarrow t^{\prime} \in \operatorname{closure}(\mathrm{C})$ and $v \preceq t^{\prime}$.

The above conditions (2) to (4) are straightforward as they follow the definition of simulation. Condition (1) follows from a property of FT-constraints: if a query node $v$ is redundant, then any descendant query node of $v$ is necessarily also redundant [14]. Since the query output node is non-redundant, all its ancestor nodes must also be non-redundant.

We use simtype $(u)$ to denote the set of types in $\Sigma$ that simulate $u$; i.e, simtype $(u)=\{t \in \Sigma \mid u \preceq t\}$. The function simtype() can be computed efficiently using two auxiliary functions partype () and anctype() which are defined as follows.

Given a set $T \subseteq \Sigma$, partype $(T)$ is defined to be the set of types in $\Sigma$ having a required-child type in $T$; and anctype $(T)$ is defined to be the set of types in $\Sigma$ having a required-descendant type in $T$ as follows:

$$
\begin{aligned}
& \operatorname{partype}(T)=\left\{t \in \Sigma \mid t \rightarrow t^{\prime} \in \operatorname{closure}(C), t^{\prime} \in T\right\} ; \\
& \operatorname{anctype}(T)=\left\{t \in \Sigma \mid t \rightarrow t^{\prime} \in \operatorname{closure}(C), t^{\prime} \in T\right\} .
\end{aligned}
$$

Based on partype() and anctype(), simtype( $u$ ) can be defined as follows: if $u$ is the output query node or its ancestor, then $\operatorname{simtype}(u)=\emptyset$; otherwise,

$$
\begin{aligned}
& \text { simtype }(u)=\{t \in \Sigma \mid t \leq \tau(u) \in \operatorname{closure}(C), \\
& \quad t \in \operatorname{partype}(\operatorname{simtype}(v)) \text { for each pc-child } v \text { of } u, \\
& \quad t \in \operatorname{anctype}(\operatorname{simtype}(v)) \text { for each } a d \text {-child } v \text { of } u\} .
\end{aligned}
$$

Node simulators. Recall that $\operatorname{sim}(u)$ denotes the set of forward simulators of $u$. The function $\operatorname{sim}()$ can be defined in terms of two additional auxiliary functions, namely, augpar(), and auganc(), which are defined as follows:

- $\operatorname{augpar}(\operatorname{sim}(u))=\operatorname{par}(\operatorname{sim}(u)) \cup\{v \in V \mid \tau(v) \in$ partype(simtype $(u))\}$; and

- $\operatorname{auganc}(\operatorname{sim}(u))=\operatorname{anc}(\operatorname{sim}(u)) \cup S_{R A} \cup \operatorname{anc}\left(S_{R A}\right)$, where $S_{R A}=\{v \in V \mid \tau(v) \in \operatorname{anctype}(\operatorname{simtype}(u))\}$.

Based on augpar() and auganc(), $\operatorname{sim}(u)$ can now be defined in terms of three cases as follows:

- if $u$ is the output node, then $\operatorname{sim}(u)=\{u\}$;

- if $u$ is a non-output leaf node, then $\operatorname{sim}(u)=\{v \in V \mid \tau(v) \leq$ $\tau(u) \in$ closure $(C)\}$;

- if $u$ is neither the output node nor a leaf node, then

$$
\begin{aligned}
& \operatorname{sim}(u)=\{v \in V \mid \tau(v) \leq \tau(u) \in \operatorname{closure}(C), v \in \\
& \quad \operatorname{augpar}\left(\operatorname{sim}\left(u^{\prime}\right)\right) \text { for each } p c \text {-child } u^{\prime} \text { of } u, \text { and } v \in \\
& \text { auganc } \left.\left(\operatorname{sim}\left(u^{\prime \prime}\right)\right) \text { for each } a d \text {-child } u^{\prime \prime} \text { of } u\right\} .
\end{aligned}
$$

Example 7.1 Consider query $Q$ shown in Figure 11(a) with constraints $C=\{b \leq B, b \rightarrow d, f \leq F, a \rightarrow f, f \rightarrow c\}$. We have $\operatorname{sim}(d)=\{d\}, \operatorname{sim}\left(e_{1}\right)=\left\{e_{1}, e_{2}\right\}, \operatorname{augpar}(\operatorname{sim}(d))=\{B, b\}$, $\operatorname{par}(\operatorname{sim}(d))=\{B\}$, and augpar $\left(\operatorname{sim}\left(e_{1}\right)\right)=\{B, b\}$. Since $b \in \operatorname{augpar}(\operatorname{sim}(d))$ and $b \in \operatorname{augpar}\left(\operatorname{sim}\left(e_{1}\right)\right)$, and $b \leq B$, we have $b \in \operatorname{sim}(B)$. We also have $\operatorname{sim}(c)=\{c\}, \operatorname{augpar}(\operatorname{sim}(c))$ $=\{F\}, \operatorname{simtype}(F)=\{f\}$ and $\operatorname{partype}(\operatorname{simtype}(F))=\{a\}$.

Algorithm MinimizeQuery-FT minimizes a given TPQ $Q$ in the presence of a set $C$ of FT-constraints. It first computes a reduced query $R(Q)$, which is the resultant query of $Q$ after repeatedly removing a leaf node that is redundant due to $\operatorname{closure}(C)$ until

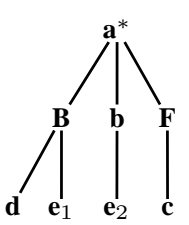

(a) $Q$

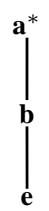

(b) $Q_{m}$

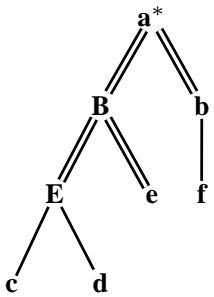

(c) $Q^{\prime}$

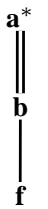

(d) $Q_{m}^{\prime}$
Figure 11: Minimization with FST-constraints

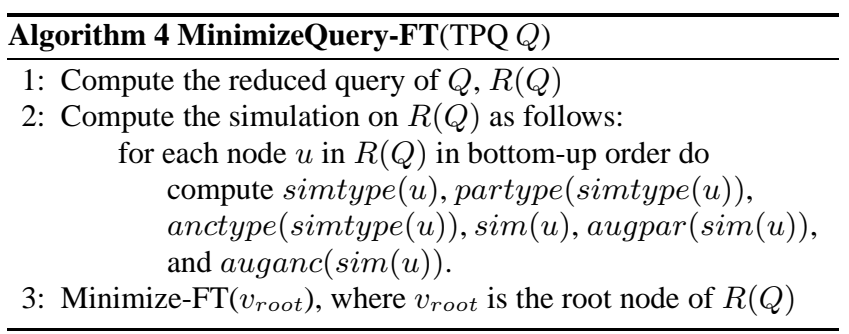

no leaf is redundant [14]. As defined in [14], a $p c$-leaf node $v$ (with parent $u$ ) of $Q$ is redundant if $\tau(u) \rightarrow \tau(v) \in$ closure $(C)$; an adleaf node $v$ (with parent $u$ ) of $Q$ is redundant if $\tau(u) \rightarrow \tau(v) \in$ closure $(C) . R(Q)$ can be computed in $O\left(n^{2}\right)$ time and has at most $n$ nodes.

Algorithm MinimizeQuery-FT then computes the type simulators and node simulators. Then it calls the recursive algorithm Minimize-FT to remove the redundant nodes as follows.

For a non-redundant node $u$ starting from the root of $R(Q)$,

- a $p c$-child $v$ of $u$ is redundant if $u$ has another $p c$-child $w \in$ $\operatorname{sim}(v)$ or $\tau(u) \in$ partype $(\operatorname{simtype}(v))$;

- an ad-child $v$ of $u$ is redundant if $u$ has another child $w \in$ $\operatorname{sim}(v) \cup \operatorname{auganc}(\operatorname{sim}(v))$ or $\tau(u) \in \operatorname{anctype}(\operatorname{simtype}(v))$.

If $v$ is redundant, the subtree rooted at $v$ is deleted. Otherwise, Minimize-FT continues minimization on $v$.

Example 7.2 (continued from Example 7.1) The $p c$-child of $a, B$, is redundant as $a$ has another $p c$-child $b \in \operatorname{sim}(B)$. Thus the subtree rooted at $B$ is deleted. At the same time, we have $F$ is redundant as $a \in$ partype(simtype $(F))$. Thus the subtree rooted at $F$ is also deleted, leading to the minimal query $Q_{m}$ as shown in Figure 11(b).

Complexity of MinimizeQuery-FT Computing the reduced query $R(Q)$ takes $O\left(n^{2}\right)$ time. The simulation relations are computed in $O(n|\Sigma|)$ time. Minimize-FT $\left(v_{\text {root }}\right)$ takes $O\left(n^{2}\right)$ time. Hence the time-complexity of MinimizeQuery-FT is $O\left(n|\Sigma|+n^{2}\right)$.

For MinimizeQuery-FT, we have the following result.

LEMma 7.1. Algorithm MinimizeQuery-FT correctly generates the minimal query for a given $T P Q Q$ in the presence of $F T$-constraints in $O\left(n|\Sigma|+n^{2}\right)$ time.

\subsection{Extensions for FST-constraints}

The minimization algorithms for FST-constraints are equivalent to those for FT-constraints except that we need to use extended definitions of both augpar() and auganc() to take into account of sibling constraints. 


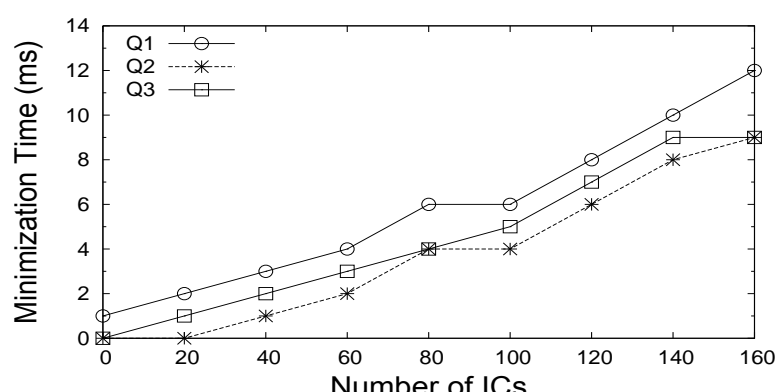

Number of ICs
(a) Varying Number of ICs

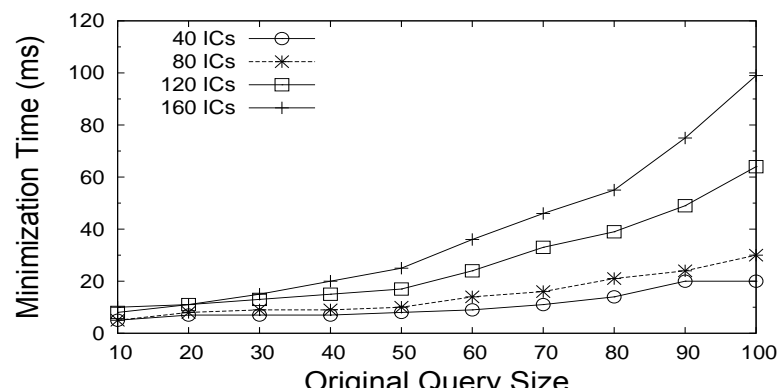

(b) Vriginal Query Size

Figure 12: Experimental Results: Minimization Time

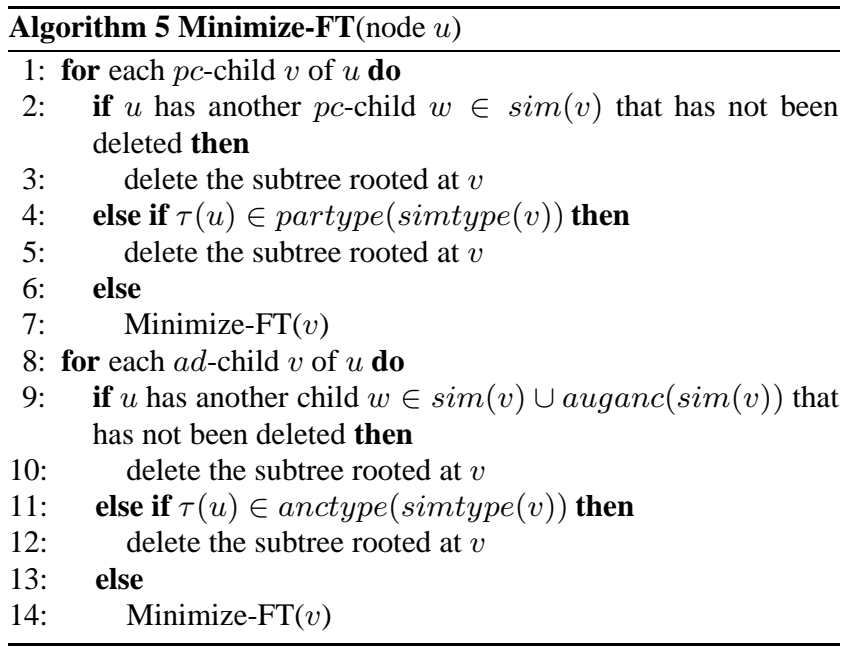

If $v$ has a $p c$-child $w$ and $\tau(v) \stackrel{\tau(w)}{\rightarrow} t \in \operatorname{closure}(C)$, then $v$ must necessarily have another $p c$-child of type $t$. When sibling constraints are included in $C$, the following extensions are needed:

- $\operatorname{augpar}(\operatorname{sim}(u))=\operatorname{par}(\operatorname{sim}(u)) \cup\{v \in V \mid \tau(v) \in$ partype $($ simtype $(u)) \cup P\}$;

- $\operatorname{auganc}(\operatorname{sim}(u))=\operatorname{anc}(\operatorname{sim}(u)) \cup S_{R A} \cup \operatorname{anc}\left(S_{R A}\right) \cup P$ $\cup \operatorname{anc}(P)\}$

where $S_{R A}=\{v \in V \mid \tau(v) \in$ anctype $($ simtype $(u))\}$; and $P$ $=\{v \in V \mid v$ has a $p c$-child $w, \tau(v) \stackrel{\tau(w)}{\rightarrow} t \in \operatorname{closure}(C), t \in$ simtype $(u)\}$.

Similarly, we have the following result.

LEMMA 7.2. In the presence of FST-constraints, a TPQ $Q$ can be minimized in $O\left(n|\Sigma|+n^{2}\right)$ time.

Example 7.3 Consider minimization of $Q^{\prime}$ in Figure 11(c) with constraints $C=\left\{b \leq B, e \leq E, e \rightarrow c, e \rightarrow d, b_{-\rightarrow}^{f} \rightarrow e\right\}$. $Q$ itself is a reduced query. We have $\operatorname{sim}(c)=\{c\}, \operatorname{sim}(d)=\{d\}$, and simtype $(c)=\{c\}, \operatorname{simtype}(d)=\{d\} ;$ and augpar $(\operatorname{sim}(c))$ $=\{E, e\}$, augpar $(\operatorname{sim}(d))=\{E, e\}$; we have $\operatorname{sim}(E)=\{E, e\}$, $\operatorname{sim}(e)=\{e\}$, and simtype $(E)=\operatorname{simtype}(e)=\{e\}$. We have $\operatorname{auganc}(\operatorname{sim}(E))=\{B, a, b\}$ and $\operatorname{auganc}(\operatorname{sim}(e))=\{B, a$, $b\}$ due to the extensions of definitions of augpar () and auganc(): $b$ has a $p c$-child $f$ such that $b \stackrel{f}{\rightarrow}$ e holds where $e \in \operatorname{simtype}(E)$ ( $e \in \operatorname{simtype}(e)$ too). Then we have $b \in \operatorname{sim}(B)$. The subtree rooted at $B$ is then deleted, which leads to minimal query $Q_{m}^{\prime}$ as shown in Figure 11(d).

\section{OTHER MINIMIZATION ALGORITHMS}

In this section, we summarize the time complexity results for computing a single minimal query for the remaining classes of constraints shown in Figure 2(b). For FBT-constraints, the time complexity is the same as that for FBST-constraints.

However, for FB/FBS-constraints, the time computing reduces to $O\left(n^{4}\right)$. The improvement in complexity is due to an optimized chase procedure: during step $\mathrm{S} 1$ of the chase procedure, only the types that exist in the query prior to chase are attached. This leads to a smaller chase query $Q_{C}$ with $O\left(n^{2}\right)$ nodes and $O\left(n^{3}\right)$ edges. Consequently, the simulation relations can be computed in $O\left(n^{4}\right)$ time.

\section{EXPERIMENTAL RESULTS}

In this section, we present an experimental study on the efficiency and effectiveness of our minimization algorithms. The algorithms being compared include both of our proposed algorithms SingleMinimizeFBST (FBST for short) and MultipleMinimizeFBST (MFBST for short), as well as CTPQMinimize (CTPQ for short) proposed in [14] for forward constraints. We used both real (DBLP records [11]) as well as synthetic (XMark [15]) datasets in our experiments. Our experimental results on both the XMark and DBLP datasets show the efficiency and scalability of FBST. All the algorithms were implemented in Java, and the experiments were run on a Pentium $4 \mathrm{PC}$ with a $3.0 \mathrm{Ghz}$ processor, 1 GB of main memory, and a 30 GB hard disk.

\subsection{Efficiency and Scalability of Minimization}

In our first set of experiments, we study the effect of the number of constraints and the effect of query size on the query minimization time of FBST. This metric measures the time to generate a minimal query for an input query. Our experimental results on both the XMark and DBLP datasets show the efficiency and scalability of FBST; however, due to space limitation, we only present the experimental results on the DBLP dataset.

For the DBLP dataset of $127 \mathrm{MB}$, we extracted 160 non-trivial constraints (29 forward, 10 backward and 121 sibling constraints). The purpose of using such a large number of constraints is to evaluate the scalability of our algorithms; the minimal queries, however, can actually be obtained using a set of no more than 20 constraints. 


\begin{tabular}{|l|l|}
\hline Input query & Minimal query produced by FBST \\
\hline \hline $\mathrm{X} 1:$ //site/people/person[name]//profile//education & //education \\
$\mathrm{X} 2: / /$ site/open_auctions/open_auction/bidder/increase & //increase \\
$\mathrm{X} 3: / /$ site/open_auctions/open_auction/reserve & //reserve \\
\hline
\end{tabular}

Table 2: Queries in comparison with CTPQMinimize

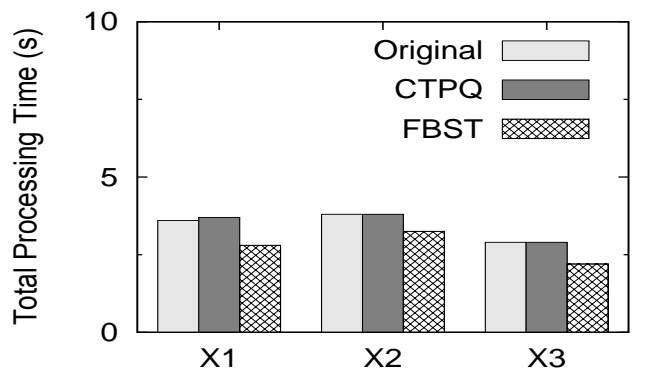

(a) XMark Dataset (111MB)

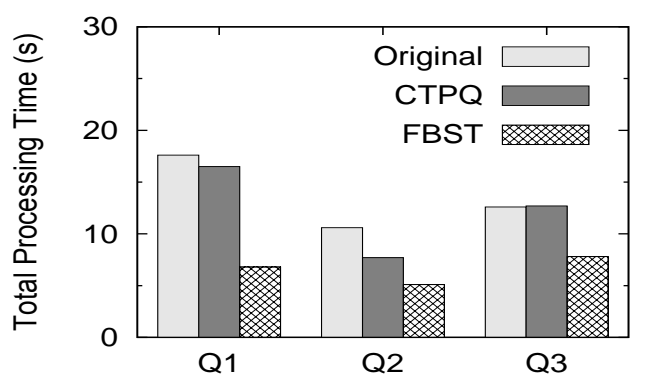

(c) DBLP Dataset(127MB)

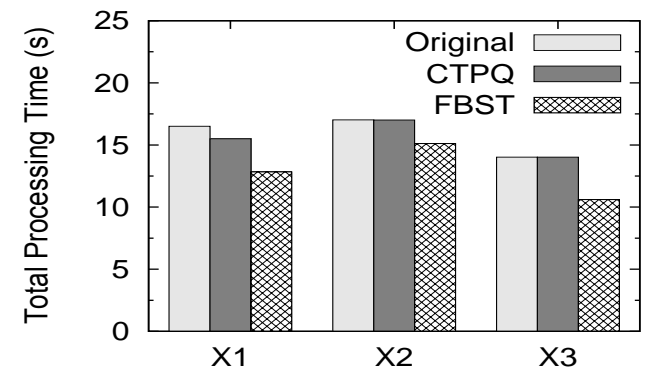

(b) XMark Dataset (384MB)

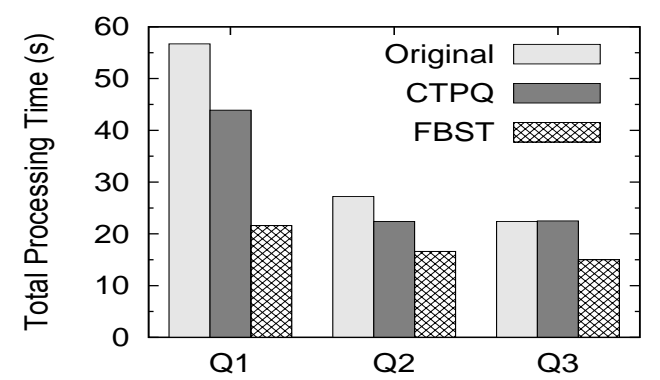

(d) DBLP Dataset(411MB)

Figure 13: Experimental Results: Total Processing Time (Minimization + Evaluation)

Effect of \#ICs on minimization time. This experiment studies the effect of the number of ICs on the minimization time of FBST for the following three queries by varying the input set of non-trivial constraints used:

$Q 1$ : //dblp/inproceedings[booktitle][year][author][url][crossref][number][pages]/title $Q 2$ : //dblp/article[volume][author][url][title][year][journal]/cite

Q3: //dblp/book[cite][author][year][isbn][publisher][title]

The sizes of $Q 1, Q 2$, and $Q 3$ are 10,9 , and 8 nodes, respectively, and their minimal queries (w.r.t. 160 ICs) have 3, 2, and 2 nodes respectively $\left(Q_{\min 1}=/ /\right.$ inproceedings//[number $] /$ ittle, $Q_{\min 2}=/ /$ article/cite, $Q_{\min 3}=/ /$ book $[$ cite $]$ ).

The results shown in Figure 12(a) indicate that the minimization time increases with the number of ICs (as expected). Note that the minimization times reported are rather small: the minimization time for $Q 1, Q 2$, and $Q 3$ (w.r.t. $160 \mathrm{ICs}$ ) is only $12 \mathrm{~ms}, 9 \mathrm{~ms}$, and $9 \mathrm{~ms}$ respectively; which is a negligible overhead in a typical XPath query evaluation process.

Effect of query size on minimization time. This experiment studies the effect of the query size on the minimization time of FBST.

We generated a set of 10 test queries of increasing sizes from 10 to 100 nodes. The 10 test queries are composed using a collection of five query fragments $\left(Q_{a}\right.$ through $\left.Q_{e}\right)$ together with a query root fragment $Q_{o}=/ / d b l p$. Note that $Q_{a}$ has 9 nodes, and each of $Q_{b}$ through $Q_{e}$ has 10 nodes.
$Q_{a}:$ phdthesis[ee][series][title][year][school][publisher][author][number]

$Q_{b}$ : proceedings[cite][journal][volume][number][publisher][editor][isbn][series]/title $Q_{c}$ : inproceedings[title][booktitle][year][number][cdrom][author][pages][url]/crossref $Q_{d}$ : incollection[chapter][isbn][publisher][ee][url][pages][author][cite]/booktitle $Q_{e}$ : book[url][isbn][title][month][year][cdrom][cite][publisher]/author

The first test query $Q_{10}$, which consists of 10 nodes, is composed using $Q_{o}$ and $Q_{a}$; and the remaining 9 test queries are composed by appending query fragments from $\left\{Q_{b}, \cdots Q_{e}\right\}$ to $Q_{10}$ under $Q_{o}$ as branches repeatedly. For each test query, we measure its minimization time w.r.t. 4 different collections of ICs consisting of $40,80,120$, and 160 constraints, respectively.

Figure 12(b) shows the minimization time for the 10 test queries w.r.t. the 4 sets of ICs. As expected, the minimization time (w.r.t. a given set of ICs) increases with a larger query size. The results also demonstrate the scalability of FBST algorithm: the minimization time for the largest test query (with 100 nodes) w.r.t. the largest set of $160 \mathrm{ICs}$ is only $99 \mathrm{~ms}$. We note that for each of the test queries, the size of its minimal query (w.r.t. to the complete set of ICs) is no more than $30 \%$ of the size of the original query.

We also conducted experiments comparing the performance of FBST and MFBST on both DBLP and XMark datasets. Our results indicate that the minimization time of MFBST (to enumerate all minimal queries) is no more than $10 \%$ longer than the minimization time of FBST (to compute a single minimal query). 


\subsection{Comparison of Total Processing Time}

In our second set of experiments, we compare the total query processing time of algorithm FBST and CTPQ. This metric measures both the time taken to generate a minimal query for an input query as well as the time taken to evaluate the minimized query against a dataset. We also compare the total processing time of FBST and CTPQ with the evaluation time of the original query without minimization.

We first ran both FBST and CTPQ to minimize the input queries on both XMark and DBLP datasets, and then evaluated the respective minimized queries on the XML datasets using the efficient query evaluation engine, GCX [16].

The test queries on XMark are shown in the first column of Table 2. Queries $X 2$ corresponds to XMark's benchmark queries Q2; while $X 1$ and $X 3$ are slightly modified versions of XMark's benchmark queries $Q 1$ and $Q 4$. As there are no benchmark queries for DBLP, we used the same synthetic queries $Q 1, Q 2$ and $Q 3$ as in Section 9.1.

The constraints used for query minimization are derived from the XMark DTD and DBLP datasets. The minimized queries produced by FBST for $X 1, X 2$ and $X 3$ are shown in the second column of Table 2. The minimal queries of $X 1, X 2$ and $X 3$ produced by CTPQ are the same as their input queries except for query $X 1$, of which the minimized query is //site/people/person//profile//education. Comparing the minimized queries produced by FBST and CTPQ, it is clear that using additional backward and sibling constraints are effective for query minimization.

In terms of query minimization time, the minimization time required by both CTPQ and FBST is less than $10 \mathrm{~ms}$ for each of the test queries. Thus, the overhead incurred by FBST using more constraints is negligible.

The minimized queries of $X 1$ through $X 3$, and $Q 1$ through $Q 3$ were then evaluated to measure their evaluation times on the XML datasets. We used two datasets of different sizes for both XMark and DBLP. For XMark, datasets of 111MB (the standard XMark dataset [15]) and 384MB (generated using XMark data generator [15]) were used; for DBLP, the $127 \mathrm{MB}$ dataset [11] and the $411 \mathrm{MB}$ dataset [2] were used.

Figure 13 compares compare the total processing time for the input queries: Original refers to the non-minimized original query, $C T P Q$ refers to the query minimized using $\mathrm{CTPQ}$, and $F B S T$ refers to the query minimized using FBST. Essentially, the minimization overhead is negligible compared to the gain in total query processing time. FBST gives the best performance compared to both CTPQ and Original. Our experimental results demonstrate the effectiveness and efficiency of TPQ minimization using a richer class of constraints.

\section{CONCLUSION}

In this paper, we examined the problem of tree pattern query minimization using a richer class of integrity constraints (FBST constraints) that includes not only forward and subtype constraints but also backward and sibling constraints. Our study revealed several interesting properties about minimal queries under FBST constraints that makes the query minimization problem more challenging. First, there can be multiple minimal queries of different sizes for an input query; and second, a minimal query can include element types that are not present in the input query. We have characterized the properties of minimal queries for various subclasses of FBST constraints, and have developed efficient algorithms, based on these properties, to both compute a single minimal query as well as enumerate all minimal queries. In addition, we have also de- veloped more efficient minimization algorithms for the previously studied class of FT constraints. Our experimental study demonstrated the effectiveness and efficiency of query minimization using FBST constraints.

\section{REPEATABILITY ASSESSMENT RESULT}

All the results in the submitted paper were verified by the SIGMOD repeatability committee. However, Figures 12 (a)\&(b) in the proceedings version of this paper include additional results that have not been verified.

Acknowledgements This research is supported in part by NUS Grant R-252-000-271-112.

\section{REFERENCES}

[1] The protein sequence database mark-up language. http://pir.georgetown.edu/pirwww/xml/psdml.dtd.

[2] The DBLP XML records. http://dblp.uni-trier.de/xml/.

[3] The Mondial database. http://www.dbis.informatik.uni-goettingen.de/Mondial/.

[4] S. Abiteboul, P. Buneman, and D. Suciu. Data on the web: from relations to semistructured data and XML. Morgan Kaufman, 2000.

[5] S. Amer-Yahia, S. Cho, L. V. S. Lakshmanan, and D. Srivastava. Minimization of tree pattern queries. In SIGMOD, 2001.

[6] G. J. Bex, F. Neven, T. Schwentick, and K. Tuyls. Inference of concise DTDs from XML data. In VLDB, 2006.

[7] U. Brandes, M. Eiglsperger, I. Herman, M. Himsolt, and M. Marshall. GraphML progress report: Structural layer proposal. LNCS, 2002.

[8] S. Flesca, F. Furfaro, and E. Masciari. On the minimization of XPath queries. In $V L D B, 2003$.

[9] G. Gottlob, C. Koch, and R. Pichler. Efficient algorithms for processing XPath queries. TODS, 30(2), 2005.

[10] S. Hinkelman. Business integration - information conformance statements (bi-ics). IBM, 2005.

[11] G. Miklau. The XML data repository. 2002. http://www.cs.washington.edu/research/xmldatasets/.

[12] G. Miklau and D. Suciu. Containment and equivalence for a fragment of XPath. JACM, 51(1), 2004.

[13] F. Neven and T. Schwentick. On the complexity of XPath containment in the presence of disjunction, DTD, and variables. $L M C S$, 2(3), 2006.

[14] P. Ramanan. Efficient algorithms for minimizing tree pattern queries. In SIGMOD, 2002.

[15] A. Schmidt, F. Waas, M. Kersten, D. Florescu, and I. Manolescu. The XML benchmark project. Technical Report INS-R0103, CWI, Netherlands, 2001.

[16] M. Schmidt, S. Scherzinger, and C. Koch. Combined static and dynamic analysis for effective buffer minimization in streaming XQuery evaluation. In ICDE, 2007.

[17] W3C. XML path language (XPath). 1999. http://www.w3.org/TR/xpath.

[18] W3C. XQuery 1.0: An XML query language. 2001. http://www.w3.org/TR/xquery.

[19] P. T. Wood. Minimising simple XPath expressions. In WebDB, 2001.

[20] P. T. Wood. Containment for XPath fragments under DTD constraints. In ICDT, 2003. 